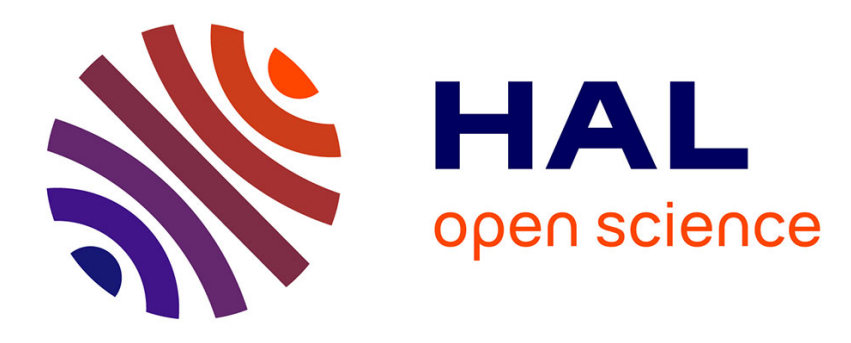

\title{
Numerical approximation of collisional plasmas by high order methods
}

Nicolas Crouseilles, Francis Filbet

\section{To cite this version:}

Nicolas Crouseilles, Francis Filbet. Numerical approximation of collisional plasmas by high order methods. Journal of Computational Physics, 2004, 201, pp.546-572. hal-00139689

\section{HAL Id: hal-00139689 \\ https://hal.science/hal-00139689}

Submitted on 3 Apr 2007

HAL is a multi-disciplinary open access archive for the deposit and dissemination of scientific research documents, whether they are published or not. The documents may come from teaching and research institutions in France or abroad, or from public or private research centers.
L'archive ouverte pluridisciplinaire HAL, est destinée au dépôt et à la diffusion de documents scientifiques de niveau recherche, publiés ou non, émanant des établissements d'enseignement et de recherche français ou étrangers, des laboratoires publics ou privés. 


\title{
Numerical approximation of collisional plasmas by high order methods
}

\author{
Nicolas Crouseilles* $\quad$ Francis Filbet ${ }^{\dagger}$
}

January 8, 2004

\begin{abstract}
In this paper, we investigate the approximation of the solution to the Vlasov equation coupled with the Fokker-Planck-Landau collision operator using a phase space grid. On the one hand, the algorithm is based on the conservation of the flux of particles and the distribution function is reconstructed allowing to control spurious oscillations and preserving positivity and energy. On the other hand, the method preserves the main properties of the collision operators in order to reach the correct stationary state. Several numerical results are presented in one dimension in space and three dimensions in velocity.
\end{abstract}

Keywords: Landau-Fokker-Planck equation, Vlasov-Poisson system, finite difference methods

\section{Contents}

1 Introduction $\quad 2$

2 Description of the kinetic model $\quad 4$

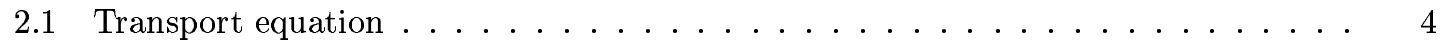

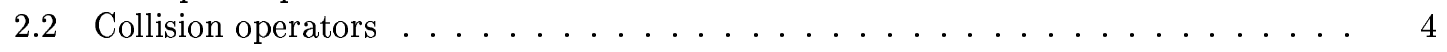

3 The numerical method $\quad 6$

3.1 Approximation to the Vlasov-Poisson system . . . . . . . . . . . . . . . . 6

3.2 Approximation to the linear operator . . . . . . . . . . . . . . . . . . . . . . . . . . . . . . . .

3.3 Approximation to the Landau operator . . . . . . . . . . . . . . . 15

4 Numerical simulations $\quad 16$

4.1 Numerical results of the linear collision operator . . . . . . . . . . . . . . 16

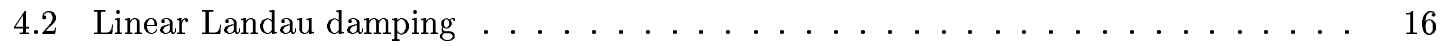

4.3 Nonlinear Landau damping . . . . . . . . . . . . . . . . . . . . . 21

5 Conclusion

*Mathématiques pour l'Industrie et la Physique, CNRS UMR 5640, Université Paul Sabatier - Toulouse 3, 118 route de Narbonne, F-31062 Toulouse cedex 4, France and CEA-CESTA (DEV/SIS), BP2 33114 Le Barp, France.crouseilles@mip.ups-tlse.fr

†Mathématiques et Applications, Physique Mathématique d'Orléans, CNRS UMR 6628, Université d'Orléans, B.P. 6759, F-45067 Orléans cedex 2, France. filbet@labomath.univ-orleans.fr 


\section{Introduction}

The evolution of a collisional plasma constituted of different species of particles is commonly described by the Landau or Fokker-Planck-Landau (FPL) equation at the kinetic level (see [10, $13,15,16,35])$. It describes binary collisions between charged particles with long-range Coulomb interactions. The evolution of particles $\alpha$ is given by the distribution function $f_{\alpha}(t, x, v)$, which depends on time $t$, position $x \in \Omega \subset \mathbb{R}^{3}$ and velocity $v \in \mathbb{R}^{3}$. This distribution function is solution to the scaled Fokker-Planck-Landau equation

$$
\frac{\partial f_{\alpha}}{\partial t}+v \cdot \nabla_{x} f_{\alpha}+E \cdot \nabla_{v} f_{\alpha}=\nu \sum_{\beta} Q_{\alpha, \beta}\left(f_{\alpha}, f_{\beta}\right)
$$

where $f_{\beta}$ is the distribution function for particles $\beta, E=E(t, x)$ is the self-consistent electric field given by the Poisson equation

$$
\nabla_{x} \cdot E(t, x)=\sum_{\beta} \int_{\mathbb{R}^{3}} f_{\beta}(v) d v, \quad E(t, x)=-\nabla_{x} \phi(t, x)
$$

where $\phi=\phi(t, x)$ represents the electric potential. Finally, $\nu$ is a nonnegative constant related to the collision frequency, $Q_{\alpha, \beta}\left(f_{\alpha}, f_{\beta}\right)$ describes $\alpha-\beta$ collisions and reads

$$
Q_{\alpha, \beta}\left(f_{\alpha}, f_{\beta}\right)=\nabla_{v} \cdot\left(\int_{\mathbf{R}^{3}} \Phi\left(v-v^{\prime}\right)\left(\nabla_{v} f_{\alpha}(v) f_{\beta}\left(v^{\prime}\right)-\nabla_{v^{\prime}} f_{\beta}\left(v^{\prime}\right) f_{\alpha}(v)\right) d v^{\prime}\right),
$$

where $\Phi(v)$ is the $3 \times 3$ matrix

$$
\Phi(v)=\frac{1}{|v|^{3}} S(v)
$$

and

$$
S(v)=|v|^{2} I_{3}-v \otimes v .
$$

Classically, the collision operator (1.3) is obtained as a remedy to the loss of finiteness of Boltzmann collision operator for long-range Coulomb interactions. In Coulomb collisions, small angle collisions play a more important role than collisions resulting in large velocity changes. The original derivation of the equation based on this idea is due to Landau [24]. Several mathematical derivations of the equation have been performed; we mention here the works of Arsen'ev and Buryak [1], Degond and Lucquin-Desreux [14], Desvillettes [17] and Rosenbluth, MacDonald and Judd [33]. For a recent review of the main mathematical aspects related to the equation, we refer the reader to Villani [35] and the references therein.

In contrast with the Boltzmann equation where Monte Carlo methods play a major role in numerical simulations, the application of these methods to long-range forces is challenging and has not yet been completely successful. Most of the particle methods for Coulomb interaction, although extensively used, have been derived more on a physical intuition basis and not directly from the Landau equation. A detailed discussion about this is beyond the aims of the present paper and we refer the reader to [29] for a more complete treatment.

Many different deterministic numerical schemes have been considered to Fokker-Planck type equations $[2,5,6,7,15,18,25,30,32]$. Due to the computational complexity of the equation (essentially caused by the large number of variables and the three-fold collision integral), many papers have been devoted to treat simpler space homogeneous situations (the distribution function $f_{\alpha}$ does not depend on $x$ ) in the isotropic case [6] or for cylindrically symmetric problems in [26]. The construction of conservative and entropic schemes for the space homogeneous case has been proposed by Degond and Lucquin-Desreux in [14] and by Buet and Cordier [5, 6]. These schemes are built in such a way that the main physical properties are conserved at a discrete level. Positivity of the solution and discrete entropy inequality are also satisfied. 
Unfortunately, the direct implementation of such schemes for space non homogeneous computations is very expensive. Indeed, the computational cost increases roughly in proportion to the square of the number of parameters used to represent the distribution function in the velocity space. Thus several fast approximated algorithms to reduce the computational complexity of these methods, based on multipole expansions [25] or multigrid techniques [7] have been proposed. A different approach, based on spectral methods, has been recently proposed for the Landau equation $[21,30]$. A detailed comparison of the spectral scheme with the schemes proposed in $[7,25]$ has been performed in [8].

Most of these methods have proven their efficiency in the homogeneous case, but few results are available in the non homogeneous situation (see [30] for some results in $2 \mathrm{D}$ velocity). The main goal of this paper is to develop a scheme in the $x$ depending case and in the whole $3 \mathrm{D}$ velocity space.

The first step consists to construct a good approximation of the Vlasov equation, which is the left hand side of (1.1). Its numerical resolution is often performed through particle methods (Particle In Cell) where the plasma is approached by a finite number of macro-particles. The trajectories of these particles are computed using the characteristic curves given by the Vlasov equation, whereas the self-consistent electric field is computed on a fixed grid (see [3] for more details). Even if these methods give satisfying results, it is well known that particle methods are noisy. Consequently, methods which discretize the Vlasov equation on a phase space grid have been proposed [19, 20,34]. These approaches allow to get an accurate approximation of the distribution function in the phase space, but the non conservation of the energy due to the projection on the grid and interpolation can be an inconvenient for the long time behavior of the solution, or for the approximation of stationary states. In this paper, we propose a new scheme using a phase space grid which overcomes these inconvenients: space and velocity derivatives are approximated by a centered finite volume method and an adapted approximation of the electric field $E$ allows us to obtain a numerical scheme that conserves the total energy. As this kind of discretization does not ensure the positivity of the unknown, we introduce slope correctors. The distribution function is reconstructed following the second order PFC method (see [19, 21]). When the slope correctors act (to avoid negative values for $f$ ) the total energy is not conserved any more; nevertheless the variations are very small compared to others methods (semi-Lagrangian method, methods using the characteristic curves).

Finally, we deal with the approximation of the collision operators $Q_{\alpha, \beta}$ given by (1.3). We first perform some assumptions on the different species of particles to get a simpler collision operator when $\alpha$ differs from $\beta$. Then, we propose a conservative scheme, based on the ideas of [15] to approximate this new operator, where particles are always interacting by Colombian potentials. For $\alpha$ - $\alpha$ interactions, the full Fokker-Planck-Landau collision operator is used. The construction of a conservative and entropic scheme for the general situation has been proposed in [15]. Even if this scheme gives interesting properties (conservations, decay of the entropy, positivity of the distribution function), its direct implementation is very expensive in high dimensions. Thus, we adopt the multigrid method used in [7] to reduce the computational cost.

The rest of the paper is organized as follows. In the next section, we draw up the main properties of the solution to the Vlasov-Poisson system coupled with Fokker-Planck-Landau collision operators and formally derive the operator intended to model collisions between different species. We then present in section 3, a finite volume scheme for the discretization of the Vlasov-Poisson equation. We next propose an approximation of the simplified collision operator and describe the discretization of the full Fokker-Planck-Landau operator. Finally, several numerical results are presented in section 4 to illustrate the efficiency of the method. 


\section{Description of the kinetic model}

\subsection{Transport equation}

We first briefly recall some classical estimates on the Vlasov-Poisson (1.1)-(1.2) system without collision (i.e. $\nu=0$ ): mass and momentum are preserved with time,

$$
\frac{d}{d t} \int_{\mathbf{R}^{3} \times \mathbf{R}^{3}} f_{\alpha}(t, x, v)\left(\begin{array}{c}
1 \\
v
\end{array}\right) d x d v=0, \quad t \in \mathbb{R}^{+} .
$$

Next, multiplying the Vlasov equation with $\nu=0(1.1)$ by $|v|^{2}$ and performing an integration by parts, we find the conservation of energy for the (1.1)-(1.2) system

$$
\frac{d}{d t}\left(\int_{\mathbf{R}^{3} \times \mathbf{R}^{3}} f_{\alpha}(t, x, v) \frac{|v|^{2}}{2} d x d v+\frac{1}{2} \int_{\mathbb{R}^{3}}|E(t, x)|^{2} d x\right)=0, \quad t \in \mathbb{R}^{+} .
$$

Finally, the Vlasov-Poisson equation (1.1)-(1.2) conserves the kinetic entropy

$$
H(t)=\int_{\mathbf{R}^{3} \times \mathbf{R}^{3}} f_{\alpha}(t) \log \left(f_{\alpha}(t)\right) d x d v=H(0) .
$$

\subsection{Collision operators}

The Fokker-Planck-Landau (FPL) operator is used for the description of binary collisions between charged particles, for which the interaction potential is the long-range Coulomb interaction. In our case, it describes electron-electron collisions. We recall the expression and some properties of the FPL collision operator

$$
Q_{\alpha, \alpha}\left(f_{\alpha}, f_{\alpha}\right)=\nabla_{v} \cdot\left(\int_{\mathbf{R}^{3}} \Phi\left(v-v^{\prime}\right)\left(\nabla_{v} f_{\alpha}(v) f_{\alpha}\left(v^{\prime}\right)-\nabla_{v^{\prime}} f_{\alpha}\left(v^{\prime}\right) f_{\alpha}(v)\right) d v^{\prime}\right),
$$

where $\Phi(v)$ is the $3 \times 3$ matrix (1.4) and can be viewed as the orthogonal projector onto the orthogonal plane to $v$. The algebraic structure of the FPL operator is similar to the Boltzmann one, this leads to physical properties such that mass, momentum and energy are conserved

$$
\int_{\mathbf{R}^{3}} Q_{\alpha, \alpha}\left(f_{\alpha}, f_{\alpha}\right)(v)\left(\begin{array}{l}
1 \\
v \\
|v|^{2}
\end{array}\right) d v=0
$$

and the the entropy $H(t)$ is decreasing,

$$
\frac{d H}{d t}(t)=\frac{d}{d t} \int_{\mathbf{R}^{3}} f_{\alpha}(t, v) \log \left(f_{\alpha}(t, v)\right) d v \leq 0 .
$$

Finally, the equilibrium state of the FPL operator, i.e. the distribution function $f_{\alpha}$ which satisfies $Q_{\alpha, \alpha}\left(f_{\alpha}, f_{\alpha}\right)=0$, is given by a Maxwellian

$$
\mathcal{M}_{\rho_{\alpha}, u_{\alpha}, T_{\alpha}}(v)=\frac{\rho_{\alpha}}{\left(2 \pi T_{\alpha}\right)^{3 / 2}} \exp \left(-\frac{\left|v-u_{\alpha}\right|^{2}}{2 T_{\alpha}}\right)
$$

where $\rho_{\alpha}$ is the total mass, $u_{\alpha}$ the mean velocity and $T_{\alpha}$ the temperature given by

$$
\rho_{\alpha}=\int_{\mathbf{R}^{3}} f_{\alpha}(v) d v, \quad u_{\alpha}=\frac{1}{\rho_{\alpha}} \int_{\mathbf{R}^{3}} f_{\alpha}(v) v d v, \quad T_{\alpha}=\frac{1}{3 \rho_{\alpha}} \int_{\mathbf{R}^{3}} f_{\alpha}(v)\left|v-u_{\alpha}\right|^{2} d v .
$$

The operator $Q_{\alpha, \beta}$ describes collisions between two different species (for instance ions and electrons) and can be derived from the two species form of the full Landau operator (1.3). If we 
assume that the temperature $T_{\beta}$ is negligeable compared to the temperature $T_{\alpha}$ of species $\alpha(2.2)$, we may consider that the distribution function of species $\beta$ is given by a Dirac measure in velocity

$$
f_{\beta}(t, x, v)=\rho_{\beta}(t, x) \delta_{0}\left(v-u_{\beta}(t, x)\right),
$$

where the density $\rho_{\beta}$ and the mean velocity $u_{\beta}$ are given or satisfy hydrodynamic equations. The so-obtained operator then reads (see [13] for a more physical derivation)

$$
Q_{\alpha, \beta}\left(f_{\alpha}\right)=\rho_{\beta} \nabla_{v} \cdot\left(\Phi\left(v-u_{\beta}\right) \nabla_{v} f_{\alpha}\right),
$$

where $\Phi(v)$ is the $3 \times 3$ matrix given by (1.4).

To emphasize the properties of $Q_{\alpha, \beta}$, it is convenient to write its weak formulation: let $\varphi$ be a smooth test function, then we have

$$
\int_{\mathbf{R}^{3}} Q_{\alpha, \beta}\left(f_{\alpha}\right)(v) \varphi(v) d v=-\rho_{\beta} \int_{\mathbf{R}^{3}}\left(\Phi\left(v-u_{\beta}\right) \nabla_{v} f_{\alpha}\right) \cdot \nabla_{v} \varphi d v .
$$

We also introduce the weak log formulation

$$
\int_{\mathbf{R}^{3}} Q_{\alpha, \beta}\left(f_{\alpha}\right)(v) \varphi(v) d v=-\rho_{\beta} \int_{\mathbf{R}^{3}}\left(\Phi\left(v-u_{\beta}\right) f_{\alpha} \nabla_{v}\left(\log f_{\alpha}\right)\right) \cdot \nabla_{v} \varphi d v .
$$

The different properties of $Q_{\alpha, \beta}$ are listed in the following proposition.

Proposition 2.1 The linear collision operator $Q_{\alpha, \beta}\left(f_{\alpha}\right)$ given by (2.4) satisfies

(i) the preservation of mass and energy, i.e.

$$
\int_{\mathbf{R}^{3}} Q_{\alpha, \beta}\left(f_{\alpha}\right)(v) d v=0, \quad \int_{\mathbf{R}^{3}} Q_{\alpha, \beta}\left(f_{\alpha}\right)(v)\left|v-u_{\beta}\right|^{2} d v=0
$$

(ii) $\operatorname{Ker}\left(Q_{\alpha, \beta}\right)=\left\{f_{\alpha}\left(\left|v-u_{\beta}\right|^{2}\right)\right\}$,

(iii) each convex function $\Psi$ of $f_{\alpha}$ is an entropy for $Q_{\alpha, \beta}$

$$
\frac{d}{d t} \int_{\mathbf{R}^{3}} \Psi\left(f_{\alpha}\right) d v \leq 0
$$

Proof. ( $i$ ) Our starting point is the weak formulation (2.5) to $Q_{\alpha, \beta}\left(f_{\alpha}\right)$. The conservation of mass easily follows taking $\varphi=1$. The conservation of energy is obtained by choosing $\varphi=\left|v-u_{\beta}\right|^{2}$ in $(2.5)$

$$
\int_{\mathbf{R}^{3}} Q_{\alpha, \beta}\left(f_{\alpha}\right)(v)\left|v-u_{\beta}\right|^{2} d v=-2 \rho_{\beta} \int_{\mathbb{R}^{3}} \nabla_{v} f_{\alpha} \cdot\left(\Phi\left(v-u_{\beta}\right)\left(v-u_{\beta}\right)\right) d v=0,
$$

because the matrix $\Phi(v)$ is symmetric and $v \in \operatorname{Ker} \Phi(v)$.

(ii) When $f_{\alpha}$ only depends on $\left|v-u_{\beta}\right|^{2}$, the following equality holds

$$
\nabla_{v}\left(f_{\alpha}\left(\left|v-u_{\beta}\right|^{2}\right)\right)=2\left(v-u_{\beta}\right) f_{\alpha}^{\prime}\left(\left|v-u_{\beta}\right|^{2}\right) .
$$

As we saw above $v \in \operatorname{Ker}(\Phi(v))$, consequently the functions $f_{\alpha}\left(\left|v-u_{\beta}\right|^{2}\right)$ vanish the collision operator.

Reciprocally, we consider $f_{\alpha} \in \operatorname{Ker}\left(Q_{\alpha, \beta}\right)$, which means that $Q_{\alpha, \beta}\left(f_{\alpha}\right)=0$. Then, we multiply $Q_{\alpha, \beta}\left(f_{\alpha}\right)$ by $f_{\alpha}$, integrate over $v \in \mathbb{R}^{3}$, and apply the Green formula

$$
\int_{\mathbf{R}^{3}} \Phi\left(v-u_{\beta}\right) \nabla_{v} f_{\alpha} \cdot \nabla_{v} f_{\alpha} d v=0 .
$$

Thus, from the nonnegativity of $\Phi(v)$ we deduce that for almost every $v \in \mathbb{R}^{3}$

$$
\Phi\left(v-u_{\beta}\right) \nabla_{v} f_{\alpha} \cdot \nabla_{v} f_{\alpha}=0 .
$$


Thanks to the fact that $\Phi(v)$ is a nonnegative matrix, (2.7) is equivalent to

$$
\nabla_{v} f_{\alpha} \in \operatorname{Ker} \Phi\left(v-u_{\beta}\right) .
$$

We know that the kernel of $\Phi(v)$ is generated by $v$, consequently (2.8) is equivalent to

$$
\nabla_{v} f_{\alpha}=\lambda(v)\left(v-u_{\beta}\right) .
$$

We conclude that $f_{\alpha}$ only depends on $\left|v-u_{\beta}\right|^{2}$ by passing to spherical coordinates in (2.9).

(iii) We introduce the following quantity $\mathcal{H}(t)$,

$$
\mathcal{H}(t)=\int_{\mathbb{R}^{3}} \Psi\left(f_{\alpha}\right)(v) d v .
$$

Then, by differentiating with respect to the time, we get

$$
\begin{aligned}
\frac{d \mathcal{H}}{d t}(t) & =\int_{\mathbf{R}^{3}} \frac{\partial f_{\alpha}}{\partial t} \Psi^{\prime}\left(f_{\alpha}\right)(v) d v \\
& =\int_{\mathbf{R}^{3}} Q_{\alpha, \beta}\left(f_{\alpha}\right)(v) \Psi^{\prime}\left(f_{\alpha}\right)(v) d v .
\end{aligned}
$$

Using (2.5) and the property of nonnegativity of the matrix $\Phi(v)$, we can easily prove

$$
\int_{\mathbf{R}^{3}} Q_{\alpha, \beta}\left(f_{\alpha}\right)(v) \Psi^{\prime}\left(f_{\alpha}\right)(v) d v=-\rho_{\beta} \int_{\mathbf{R}^{3}}\left(\Phi\left(v-u_{\beta}\right) \nabla_{v} f_{\alpha}\right) \cdot\left(\nabla_{v} \Psi^{\prime}\left(f_{\alpha}\right)\right)(v) d v .
$$

Finally, since $\Psi$ is a convex function of $f_{\alpha}$

$$
\int_{\mathbf{R}^{3}} Q_{\alpha, \beta}\left(f_{\alpha}\right)(v) \Psi^{\prime}\left(f_{\alpha}\right)(v) d v=-\rho_{\beta} \int_{\mathbf{R}^{3}}\left(\Phi\left(v-u_{\beta}\right) \nabla_{v} f_{\alpha}\right) \cdot \nabla_{v} f_{\alpha} \Psi^{\prime \prime}\left(f_{\alpha}\right)(v) d v \leq 0 .
$$

\section{The numerical method}

From now, we will only consider one species of particles, then the index $\alpha$ will be dropped and the distribution function will be denoted by $f$. We first give a finite volume scheme to the VlasovPoisson equation and then describe a conservative and entropy decreasing method to the collision operators.

\subsection{Approximation to the Vlasov-Poisson system}

We introduce in this section a new scheme, based on a finite volume method for the discretization of the Vlasov-Poisson equation. Thanks to this discretization coupled with an adapted approximation of the electric field, we will prove that this scheme preserves the total energy. We consider a cartesian grid in the phase space. For the sake of simplicity, we present the scheme in one dimension in space and velocity, but it can be easily generalized to any higher dimensions.

We introduce the mesh points $\left(x_{i+1 / 2}\right)_{i \in I}$ and $\left(v_{j+1 / 2}\right)_{j \in \mathbb{Z}}$ of the computational domain $\left[x_{\text {min }}, x_{\text {max }}\right] \times$ $\mathbb{R}$. We will denote by $\Delta x=x_{i+1 / 2}-x_{i-1 / 2}$ and $\Delta v=v_{j+1 / 2}-v_{j-1 / 2}$ the space and velocity steps and by $C_{i, j}=\left[x_{i-1 / 2}, x_{i+1 / 2}\right] \times\left[v_{j-1 / 2}, v_{j+1 / 2}\right]$ the control volume. Finally, $t^{n}=n \Delta t$ is the time discretization.

We assume that $f_{i, j}^{n}$ is an average approximation of $f$ on the control volume $C_{i, j}$ at time $t^{n}$

$$
f_{i, j}^{n}=\frac{1}{\Delta x \Delta v} \int_{C_{i, j}} f\left(t^{n}, x, v\right) d x d v
$$


Then, we approximate the average of $f$ at the time $t^{n+1}$ by integrating the Vlasov equation on a control volume and using a backward Euler scheme in time

$$
\begin{aligned}
f_{i, j}^{n+1}= & f_{i, j}^{n}-\frac{\Delta t}{\Delta x \Delta v} \int_{v_{j-1 / 2}}^{v_{j+1 / 2}} v\left(f^{n}\left(x_{i+1 / 2}, v\right)-f^{n}\left(x_{i-1 / 2}, v\right)\right) d v \\
& -\frac{\Delta t}{\Delta x \Delta v} \int_{x_{i-1 / 2}}^{x_{i+1 / 2}} \tilde{E}^{n}(x)\left(f^{n}\left(x, v_{j+1 / 2}\right)-f^{n}\left(x, v_{j-1 / 2}\right)\right) d x
\end{aligned}
$$

We have to approach the fluxes at the interface of the control volume $\left[x_{i-1 / 2}, x_{i+1 / 2}\right)$ and $\left[v_{j-1 / 2}, v_{j+1 / 2}\right)$

$$
\begin{gathered}
\frac{1}{\Delta v} \int_{v_{j-1 / 2}}^{v_{j+1 / 2}} v f^{n}\left(x_{i+1 / 2}, v\right) d v, \quad \forall i \in I, \\
\frac{1}{\Delta x} \int_{x_{i-1 / 2}}^{x_{i+1 / 2}} \tilde{E}^{n}(x) f^{n}\left(x, v_{j+1 / 2}\right) d x, \quad \forall j \in \mathbb{Z},
\end{gathered}
$$

where $\tilde{E}^{n}(x)$ is an approximation of the electric field deduced from the numerical resolution to the Poisson equation (1.2).

The main step to get an accurate solution is to reconstruct the distribution function in each direction. For that purpose, we fix the velocity $v \in\left[v_{j-1 / 2}, v_{j+1 / 2}\right)$ and consider the function $f_{h}(x, v)$ as an approximation of the distribution function $f\left(t^{n}, x, v\right)$. Thus, the high order approximation $f_{h}(x, v)$ is obtained via a reconstruction by primitive $F_{h}(x, v)$ (where $F_{h}(x, v)$ stands for the primitive of $f_{h}(x, v)$ with respect to $\left.x\right)$. We denote by

$$
f_{i}^{n}(v)=\frac{1}{\Delta x} \int_{x_{i-1 / 2}}^{x_{i+1 / 2}} f\left(t^{n}, x, v\right) d x, \text { for } v \in\left[v_{j-1 / 2}, v_{j+1 / 2}\right)
$$

hence,

$$
F_{h}\left(x_{i+1 / 2}, v\right)-F_{h}\left(x_{i-1 / 2}, v\right)=\Delta x f_{i}^{n}(v) .
$$

We present a method of reconstruction allowing to obtain a second order scheme, which preserves the positivity using slope limiters. To this aim, we first build an approximation of the primitive using the stencil $\left\{x_{i-1 / 2}, x_{i+1 / 2}, x_{i+3 / 2}\right\}$ when the velocity $v$ is positive and $\left\{x_{i-3 / 2}, x_{i-1 / 2}, x_{i+1 / 2}\right\}$ when $v$ is negative. Let us assume that $v$ is positive, then we get

$$
F_{h}(x, v)=F_{h}\left(x_{i-1 / 2}, v\right)+\left(x-x_{i-1 / 2}\right) f_{i}^{n}(v)+\frac{\left(x-x_{i-1 / 2}\right)\left(x-x_{i+1 / 2}\right)}{2 \Delta x}\left(f_{i+1}^{n}(v)-f_{i}^{n}(v)\right) .
$$

By differentiation, we obtain a second order accurate approximation of the distribution function on the interval $\left[x_{i-1 / 2}, x_{i+1 / 2}\right)$

$$
f_{h}(x, v)=\frac{\partial F_{h}}{\partial x}(x, v)=f_{i}^{n}(v)+\frac{\left(x-x_{i}\right)}{\Delta x}\left(f_{i+1}^{n}(v)-f_{i}^{n}(v)\right)
$$

This appears as a second order approximation, which can generate spurious oscillations. We then introduce a slope corrector, ensuring the positivity of the distribution function on the interval $\left[x_{i-1 / 2}, x_{i+1 / 2}\right)$

$$
f_{h}(x, v)=f_{i}^{n}(v)+\eta_{i}^{+}(v) \frac{\left(x-x_{i}\right)}{\Delta x}\left(f_{i+1}^{n}(v)-f_{i}^{n}(v)\right),
$$

with

$$
\eta_{i}^{+}(v)=\min \left(1,2 f_{i}^{n}(v) /\left(f_{i+1}^{n}(v)-f_{i}^{n}(v)\right)\right), \quad \text { if } \quad\left(f_{i+1}^{n}(v)-f_{i}^{n}(v)\right)>0 .
$$

For a negative velocity $v$, the reconstruction on the interval $\left(x_{i-1 / 2}, x_{i+1 / 2}\right)$ is

$$
f_{h}(x, v)=f_{i}^{n}(v)+\eta_{i}^{-}(v) \frac{\left(x-x_{i}\right)}{\Delta x}\left(f_{i}^{n}(v)-f_{i-1}^{n}(v)\right),
$$


with

$$
\eta_{i}^{-}(v)=\min \left(1,-2 f_{i}^{n}(v) /\left(f_{i}^{n}(v)-f_{i-1}^{n}(v)\right)\right), \quad \text { if } \quad\left(f_{i}^{n}(v)-f_{i-1}^{n}(v)\right)<0 .
$$

Now, we proceed in the same way to reconstruct $f_{h}$ in the $v$ direction depending on the sign of $\tilde{E}^{n}(x)$.

The approximation of the distribution function $f_{h}(x, v)$ given by (3.2)-(3.3) or by (3.4)-(3.5) satisfies the following properties.

Proposition 3.1 The approximation of the distribution function $f_{h}(x, v)$ defined by (3.2) or by (3.4) using the second order reconstruction with the slope correctors (3.3) and (3.5) satisfies

- The conservation of the average

$$
\frac{1}{\Delta x \Delta v} \int_{x_{i-1 / 2}}^{x_{i+1 / 2}} \int_{v_{j-1 / 2}}^{v_{j+1 / 2}} f_{h}(x, v) d x d v=f_{i, j}^{n}
$$

- The positivity of $f_{h}(x, v)$.

We finally obtain the following scheme, which represents an approximation of (3.1)

$$
f_{i, j}^{n+1}=f_{i, j}^{n}-\frac{\Delta t}{\Delta x \Delta v}\left(\psi_{i+1 / 2, j}^{n}-\psi_{i-1 / 2, j}^{n}\right)-\frac{\Delta t}{\Delta x \Delta v}\left(\phi_{i, j+1 / 2}^{n}-\phi_{i, j-1 / 2}^{n}\right),
$$

with

$$
\psi_{i+1 / 2}^{n}=\Delta v v_{j} f_{i+1 / 2, j}^{n}, \phi_{i, j+1 / 2}^{n}=\Delta x \tilde{E}_{i}^{n} f_{i, j+1 / 2}^{n},
$$

where the distribution function is approximated on the boundary of the control volume as follows

$$
f_{i+1 / 2, j}^{n}= \begin{cases}f_{i, j}^{n}+\eta_{i, j}^{+}\left(f_{i+1, j}^{n}-f_{i, j}^{n}\right) / 2, & \text { if } \quad v_{j} \geq 0 \\ f_{i+1, j}^{n}-\eta_{i+1, j}^{-}\left(f_{i+1, j}^{n}-f_{i, j}^{n}\right) / 2, & \text { if } \quad v_{j}<0\end{cases}
$$

and

$$
f_{i, j+1 / 2}^{n}=\left\{\begin{array}{lll}
f_{i, j}^{n}+\varepsilon_{i, j}^{+}\left(f_{i, j+1}^{n}-f_{i, j}^{n}\right) / 2, & \text { if } & \tilde{E}_{i}^{n} \geq 0 \\
f_{i, j+1}^{n}-\varepsilon_{i, j+1}^{-}\left(f_{i, j+1}^{n}-f_{i, j}^{n}\right) / 2, & \text { if } & \tilde{E}_{i}^{n}<0
\end{array}\right.
$$

where the slope limiters are given by

$$
\begin{array}{lll}
\eta_{i, j}^{+}=\min \left(1,2 f_{i, j}^{n} /\left(f_{i+1, j}^{n}-f_{i, j}^{n}\right)\right), & \text { if } \quad & \left(f_{i+1, j}^{n}-f_{i, j}^{n}\right)>0, \\
\eta_{i, j}^{-}=\min \left(1,-2 f_{i, j}^{n} /\left(f_{i, j}^{n}-f_{i-1, j}^{n}\right)\right), & \text { if } \quad & \left(f_{i, j}^{n}-f_{i-1, j}^{n}\right)<0,
\end{array}
$$

for the space variable, and

$$
\begin{array}{lll}
\varepsilon_{i, j}^{+}=\min \left(1,2 f_{i, j}^{n} /\left(f_{i, j+1}^{n}-f_{i, j}^{n}\right)\right), & \text { if } \quad & \left(f_{i, j+1}^{n}-f_{i, j}^{n}\right)>0, \\
\varepsilon_{i, j}^{-}=\min \left(1,-2 f_{i, j}^{n} /\left(f_{i, j}^{n}-f_{i, j-1}^{n}\right)\right), & \text { if } \quad & \left(f_{i, j}^{n}-f_{i, j-1}^{n}\right)<0,
\end{array}
$$

for the velocity variable. The following proposition gives some properties of the scheme

Proposition 3.2 Assume the initial datum $\left(f_{i, j}^{0}\right)_{i, j}$ is nonnegative. If the time step satisfies the following CFL type condition

$$
\Delta t \leq C \min (\Delta x, \Delta v)
$$

where $C>0$ is related to the maximum norm of the electric field and the upper bound of the velocity domain. Then the scheme defined by (3.6) gives a nonegative approximation. 
The proof can be deduced from the Proposition 2.1.

When the slope correctors do not occur in the approximation of the velocity derivative (it is the case when the distribution function is sufficiently smooth), we obtain a classical centered scheme

$$
\frac{1}{\Delta x \Delta v}\left(\phi_{i, j-1 / 2}^{n}-\phi_{i, j+1 / 2}\right)=\tilde{E}_{i}^{n} \frac{f_{i, j+1}^{n}-f_{i, j-1}^{n}}{2 \Delta v} .
$$

Thus, from the following approximation of the electric field

$$
\tilde{E}_{i}^{n}=\frac{E_{i}^{n+1}+E_{i}^{n}}{2}
$$

the so-obtained scheme can be written as

$$
f_{i, j}^{n+1}=f_{i, j}^{n}-\frac{\Delta t}{\Delta x \Delta v}\left(\psi_{i+1 / 2, j}^{n}-\psi_{i-1 / 2, j}^{n}\right)-\Delta t\left(\frac{E_{i}^{n+1}+E_{i}^{n}}{2} D_{c, v} f_{i, j}^{n}\right)
$$

where $D_{c, v}$ is the usual second order centered discrete operator

$$
D_{c, v} f_{j}=\frac{f_{j+1}-f_{j-1}}{2 \Delta v} .
$$

On the other hand, the electric field at time $t^{n}$ is determined through the following approximation to the Poisson equation

$$
-D_{x}^{\star} E_{i}^{n}=\rho_{i}^{n}-\rho_{\beta}, \quad E_{i}^{n}=-D_{x} \phi_{i}^{n},
$$

where $D_{x}$ is a discrete finite difference operator whereas $D_{x}^{\star}$ stands for its formal adjoint, which represents an approximation of $-\partial_{x}$ (for example, we can consider $D_{x}$ as the usual uncentered discrete operator). Hence, the following equality holds

$$
\left\langle D^{\star} \varphi, f\right\rangle=\langle\varphi, D f\rangle, \text { for all sequences } \varphi, f,
$$

where $\langle$,$\rangle denotes an inner product. Finally, E_{i}^{n+1}$ is a prediction of the electric field at time $t^{n+1}$ obtained from the discretization to the Poisson equation (3.13) and the continuity equation

$$
\rho^{n+1}=\rho_{i}^{n}+\Delta t D_{x}^{\star} J_{i}^{n},
$$

where $J_{i}^{n}$ is an approximation of the current density $j(t, x)$

$$
J_{i}^{n}=\Delta v \sum_{j \in \mathbf{Z}} v_{j} f_{i, j}^{n}
$$

whereas $\rho_{i}^{n}$ is computed iteratively from the initial density. The approximation of the VlasovPoisson equation (3.11)-(3.13) obtained from this algorithm satisfies

Proposition 3.3 The approximation of the distribution function defined by the scheme (3.11)(3.13) preserves total mass

$$
\Delta x \Delta v \sum_{i, j} f_{i, j}^{n}=\Delta x \Delta v \sum_{i, j} f_{i, j}^{0}
$$

and total energy

$$
\frac{\Delta x \Delta v}{2} \sum_{i, j}\left|v_{j}\right|^{2} f_{i, j}^{n}+\frac{\Delta x}{2} \sum_{i}\left(E_{i}^{n}\right)^{2}=\frac{\Delta x \Delta v}{2} \sum_{i, j}\left|v_{j}\right|^{2} f_{i, j}^{0}+\frac{\Delta x}{2} \sum_{i}\left(E_{i}^{0}\right)^{2} .
$$

Proof. The conservation of total mass can be obtained by multiplying (3.11) by one and summing on $i \in \mathbb{Z}, j \in \mathbb{Z}$. Then, after a discrete integration by parts, we find that mass at time $t^{n+1}$ is equal to mass at time $t^{n}$. 
For the conservation of total energy, we first introduce the following notation for the kinetic energy

$$
\mathcal{E}_{K}^{n}:=\frac{\Delta x \Delta v}{2} \sum_{i, j \in \mathbb{Z}} f_{i, j}^{n}\left|v_{j}\right|^{2}
$$

Then, if we multiply (3.11) by $\left|v_{j}\right|^{2} / 2$ and sum over $i, j \in \mathbb{Z}$, we obtain

$$
\begin{aligned}
\mathcal{E}_{K}^{n+1}= & \mathcal{E}_{K}^{n}-\frac{\Delta t}{2} \sum_{i, j \in \mathbb{Z}}\left(\psi_{i+1 / 2, j}^{n}-\psi_{i-1 / 2, j}^{n}\right)\left|v_{j}\right|^{2} \\
& -\frac{\Delta t}{2} \frac{\Delta v \Delta x}{2} \sum_{i, j \in \mathbb{Z}}\left(E_{i}^{n+1}+E_{i}^{n}\right) D_{c, v} f_{i, j}^{n}\left|v_{j}\right|^{2} .
\end{aligned}
$$

On the one hand, the space flux terms vanish thanks to a discrete integration by parts. On the other hand, we replace $E_{i}^{n}$ by $\left(-D_{x} \phi_{i}^{n}\right)$ thanks to the Poisson equation (3.13) and perform a discrete integration by parts in velocity and next in space; we obtain

$$
\begin{aligned}
\mathcal{E}_{K}^{n+1} & =\mathcal{E}_{K}^{n}+\frac{\Delta t}{4} \Delta x \Delta v \sum_{i, j \in \mathbf{Z}}\left(D_{x} \phi_{i}^{n+1}+D_{x} \phi_{i}^{n}\right) f_{i, j}^{n} D_{c, v}^{\star}\left|v_{j}\right|^{2}, \\
& =\mathcal{E}_{K}^{n}+\frac{\Delta t}{4} \Delta v \Delta x \sum_{i, j \in \mathbf{Z}}\left(\phi_{i}^{n+1}+\phi_{i}^{n}\right) D_{x}^{\star} f_{i, j}^{n} D_{c, v}^{\star}\left|v_{j}\right|^{2},
\end{aligned}
$$

where $D_{c, v}^{\star}$ stands for the adjoint of $D_{c, v}$. The centered discrete operator is a second order approximation, hence it is the exact gradient at least up to polynomial functions of degree two

$$
\begin{aligned}
\frac{\Delta v}{2} \sum_{j \in \mathbb{Z}} D_{x}^{\star} f_{i, j}^{n} D_{c, v}^{\star}\left|v_{j}\right|^{2} & =-\Delta v \sum_{j \in \mathbb{Z}} D_{x}^{\star} f_{i, j}^{n} v_{j}, \\
& =-D_{x}^{\star}\left(\Delta v \sum_{j \in \mathbb{Z}} f_{i, j}^{n} v_{j}\right)=-D_{x}^{\star} J_{i}^{n},
\end{aligned}
$$

where $J_{i}^{n}$ is the approximation of the current density (3.15). Thus, from the discrete continuity equation (3.14), we get

$$
\left.\mathcal{E}_{K}^{n+1}=\mathcal{E}_{K}^{n}-\frac{\Delta x}{2} \sum_{i \in \mathbb{Z}}\left(\phi_{i}^{n+1}+\phi_{i}^{n}\right)\right)\left(\rho_{i}^{n+1}-\rho_{i}^{n}\right) .
$$

From the approximation of the Poisson equation (3.13), we finally obtain

$$
\begin{aligned}
\mathcal{E}_{K}^{n+1} & =\mathcal{E}_{K}^{n}+\frac{\Delta x}{2} \sum_{i \in \mathbb{Z}}\left(\phi_{i}^{n+1}+\phi_{i}^{n}\right)\left(D_{x}^{\star} E_{i}^{n+1}-D_{x}^{\star} E_{i}^{n}\right), \\
& =\mathcal{E}_{K}^{n}-\frac{\Delta x}{2} \sum_{i \in \mathbb{Z}}\left(\left(E_{i}^{n+1}\right)^{2}-\left(E_{i}^{n}\right)^{2}\right) .
\end{aligned}
$$

We then conclude the total energy conservation

$$
\mathcal{E}_{K}^{n+1}+\frac{\Delta x}{2} \sum_{i \in \mathbf{Z}}\left(E_{i}^{n+1}\right)^{2}=\mathcal{E}_{K}^{n}+\frac{\Delta x}{2} \sum_{i \in \mathbb{Z}}\left(E_{i}^{n}\right)^{2}
$$

Remark 3.4 In one space dimension, the Ampère equation can be used instead of the Poisson equation. In this case, the previous demonstration is simplified. Indeed, in (3.14), $J_{i}^{n}$ can be approximated thanks to the discretized Ampère equation

$$
E_{i}^{n+1}-E_{i}^{n}=-\Delta t J_{i}^{n} .
$$

This approximation is performed in [23]. 


\subsection{Approximation to the linear operator}

This section is devoted to the discretization of the $\alpha-\beta$ collision operator. We may restrict ourselves to the space homogeneous equation and since $\rho_{\beta}$ and $u_{\beta}$ only play the role of parameters, we set

$$
\rho_{\beta}=1, \quad u_{\beta}=0 .
$$

Then, we approximate the following equation

$$
\frac{\partial f}{\partial t}=Q_{\alpha, \beta}(f),\left.f\right|_{t=0}=f_{0}(v)
$$

where $Q_{\alpha, \beta}(f)$ is given by (2.4) and $f_{0}$ stands for an initial datum. Let $v_{j}=j \Delta v$ and $j=$ $\left(j_{1}, j_{2}, j_{3}\right) \in \mathbb{Z}^{3}$ be a uniform mesh in $\mathbb{R}^{3}$ and we denote by $f_{j}$ an approximation of $f\left(v_{j}\right)$. We define $D$ as a finite difference operator that approximates the usual gradient operator $\nabla_{v}$ and by $D^{\star}$ its formal adjoint, which represents an approximation of $-\nabla_{v} \cdot$. Then, for any test sequence $\left(\varphi_{j}\right)_{j \in \mathbf{Z}^{3}}$, we set $\left(D \varphi_{j}\right)_{j \in \mathbb{Z}^{3}}$ as a sequence of vectors of $\mathbb{R}^{3}$

$$
D \varphi_{j}=\left(D^{1} \varphi_{j}, D^{2} \varphi_{j}, D^{3} \varphi_{j}\right) \in \mathbb{R}^{3},
$$

where the components $D^{s} \varphi_{j}, s \in\{1,2,3\}$ approximates the partial derivatives $\left(\partial \varphi / \partial v_{s}\right)\left(v_{j}\right)$.

To preserve the property of decreasing entropy at the discrete level, we use the log weak formulation (2.6) as in [15]. Using the notations introduced above, the operator $Q_{\alpha, \beta}(f)(v)$ can be approximated at $v=v_{j}$ by

$$
Q_{\alpha, \beta}(f)\left(v_{j}\right)=-D^{\star}\left(\frac{1}{\left|v_{j}\right|^{3}} S\left(\tilde{v}_{j}\right) f_{j} D\left(\log f_{j}\right)\right),
$$

where $S\left(\tilde{v}_{j}\right)$ is an approximation at $\tilde{v}_{j}$ to the matrix $S$ given by (1.5) i.e.

$$
S\left(\tilde{v}_{j}\right)=\left|\tilde{v}_{j}\right|^{2} I_{3}-\tilde{v}_{j} \otimes \tilde{v}_{j} .
$$

The weak formulation reads

$$
\begin{aligned}
\Delta v^{3} \sum_{j \in \mathbb{Z}^{3}} Q_{\alpha, \beta}(f)\left(v_{j}\right) \varphi_{j} & =-\Delta v^{3} \sum_{j \in \mathbb{Z}^{3}} \frac{1}{\left|v_{j}\right|^{3}} S\left(\tilde{v}_{j}\right) f_{j} D\left(\log f_{j}\right) \cdot D \varphi_{j} \\
& =-\Delta v^{3} \sum_{j \in \mathbb{Z}^{3}} \frac{1}{\left|v_{j}\right|^{3}} f_{j} D\left(\log f_{j}\right) \cdot\left(S\left(\tilde{v}_{j}\right) D \varphi_{j}\right) .
\end{aligned}
$$

We perform a discrete integration by parts and use the symmetry of $S$; we finally get the following proposition

Proposition 3.5 The discretization (3.22) conserves total mass and decreases discrete entropy

$$
\mathcal{H}(t)=\Delta v^{3} \sum_{j \in \mathbb{Z}^{3}} f_{j}(t) \log \left(f_{j}(t)\right)
$$

Moreover, it preserves energy under the condition on $\tilde{v}_{j}$

$$
D^{1}\left(\left|v_{j}\right|^{2}\right) / \tilde{v}_{j}^{1}=D^{2}\left(\left|v_{j}\right|^{2}\right) / \tilde{v}_{j}^{2}=D^{3}\left(\left|v_{j}\right|^{2}\right) / \tilde{v}_{j}^{3},
$$

where $\tilde{v}_{j}=\left(\tilde{v}_{j}^{1}, \tilde{v}_{j}^{2}, \tilde{v}_{j}^{3}\right)$.

Proof. We start from the discrete log weak formation (3.23) of $Q_{\alpha, \beta}$, for a discrete operator $D$ which stands for an approximation of the gradient operator in velocity. Then taking $\varphi=1$, it easily leads to the conservation of mass. In the same way, we take $\varphi=\log f$ in (3.22),

$$
\Delta v^{3} \sum_{j \in \mathbb{Z}^{3}} Q_{\alpha, \beta}(f)\left(v_{j}\right) \log f_{j}=-\Delta v^{3} \sum_{j \in \mathbb{Z}^{3}} \frac{1}{\left|v_{j}\right|^{3}}\left(S\left(\tilde{v}_{j}\right) f_{j} D\left(\log f_{j}\right)\right) \cdot D\left(\log f_{j}\right)
$$


which is nonpositive thanks to the semi-positivity of $S\left(\tilde{v}_{j}\right)$.

To prove the conservation of energy, we start from (3.23) taking $\varphi(v)$ equal to $|v|^{2}$

$$
\Delta v^{3} \sum_{j \in \mathbb{Z}^{3}} Q_{\alpha, \beta}(f)\left(v_{j}\right)\left|v_{j}\right|^{2}=-\Delta v^{3} \sum_{j \in \mathbb{Z}^{3}} \frac{1}{\left|v_{j}\right|^{3}} f_{j} D\left(\log f_{j}\right) \cdot\left(S\left(\tilde{v}_{j}\right) D\left|v_{j}\right|^{2}\right) .
$$

This quantity vanishes when $S\left(\tilde{v}_{j}\right) D\left|v_{j}\right|^{2}$ is zero. This $3 \times 3$ system is satisfied if and only if $\tilde{v}_{j}$ is chosen as follows

$$
D^{1}\left(\left|v_{j}\right|^{2}\right) / \tilde{v}_{j}^{1}=D^{2}\left(\left|v_{j}\right|^{2}\right) / \tilde{v}_{j}^{2}=D^{3}\left(\left|v_{j}\right|^{2}\right) / \tilde{v}_{j}^{3} .
$$

Then we conclude on the conservation of the energy.

Example 3.1 If we choose $D=D_{+}$i.e. the forward uncentered discrete operator, (3.24) implies that $\tilde{v}$ must be chosen as follows

$$
\tilde{v}_{j}^{s}=\frac{1}{2}\left(v_{j}^{s}+v_{j+e_{s}}^{s}\right), \quad s \in\{1,2,3\} .
$$

The choice of $D=D_{-}$leads to the following $\tilde{v}$

$$
\tilde{v}_{j}^{s}=\frac{1}{2}\left(v_{j}^{s}+v_{j-e_{s}}^{s}\right), \quad s \in\{1,2,3\} .
$$

Finally, if $D=D_{c}$ i.e. the centered discrete operator, (3.24) gives for $\tilde{v}$

$$
\tilde{v}_{j}^{s}=\frac{1}{2}\left(v_{j+e_{s}}^{s}+v_{j-e_{s}}^{s}\right), \quad s \in\{1,2,3\},
$$

where $e_{s}$ denotes the unit vector of the canonical basis of $\mathbb{R}^{3}, s=1,2,3$.

As mentionned in [5] and [15], the use of the centered discrete difference operator $D=D_{c}$ leads to conserved quantities which are not physical. On the other hand, the use of the uncentered discrete operator $\left(D=D_{+}\right.$or $\left.D=D_{-}\right)$introduces some unsymmetry in the distribution function leading to a loss of accuracy. To overcome these difficulties, following the idea of [5] and [7], we introduce a symmetrization of the discrete operator based on the averaging of the uncentered discretizations

$$
Q_{\alpha, \beta}(f)\left(v_{j}\right)=-\frac{1}{2}\left[D_{+}^{\star}\left(\frac{1}{\left|v_{j}\right|^{3}} S\left(\tilde{v}_{j}^{+}\right) f_{j} D_{+}\left(\log f_{j}\right)\right)+D_{-}^{\star}\left(\frac{1}{\left|v_{j}\right|^{3}} S\left(\tilde{v}_{j}^{-}\right) f_{j} D_{-}\left(\log f_{j}\right)\right)\right],
$$

where

$$
\tilde{v}_{j}^{+, s}=\frac{1}{2}\left(v_{j}^{s}+v_{j+e_{s}}^{s}\right), \text { and } \tilde{v}_{j}^{-, s}=\frac{1}{2}\left(v_{j}^{s}+v_{j-e_{s}}^{s}\right), s=1,2,3,
$$

are chosen as in Example 3.1 to conserve the energy ( $\tilde{v}^{+}$with $D_{+}$and $\tilde{v}^{-}$with $D_{-}$).

Consequently, thanks to Proposition 3.5, such a discretization conserves mass, energy and decreases the entropy. Moreover, if the mean velocity of $f$ vanishes, then the momentum of $Q_{\alpha, \beta}(f)$ is equal to zero. This property is preserved at the discrete level for the approximation (3.26) under some symmetry assumptions on the initial datum.

Proposition 3.6 We consider the discrete collision operator (3.26)-(3.27). If the sequence $\left(f_{j}\right)_{j \in \mathbb{Z}^{3}}$ is symmetric in all the directions (i.e. $f_{k}=f_{j}$, with $k_{s}=-j_{s}, s=1,2,3$ ), then the discrete collision operator (3.26)-(3.27) conserves the momentum.

Proof. We consider the $k$-th component of the momentum

$$
\begin{aligned}
\Delta v^{3} \sum_{j \in \mathbf{Z}^{3}} Q_{\alpha, \beta}(f)\left(v_{j}\right) v_{j_{k}}= & -\frac{\Delta v^{3}}{2} \sum_{j \in \mathbb{Z}^{3}} \frac{1}{\left|v_{j}\right|^{3}} \sum_{l=1}^{3} S_{k, l}\left(\tilde{v}_{j}^{+}\right) f_{j} D_{+}^{l}\left(\log f_{j}\right) \\
& -\frac{\Delta v^{3}}{2} \sum_{j \in \mathbb{Z}^{3}} \frac{1}{\left|v_{j}\right|^{3}} \sum_{l=1}^{3} S_{k, l}\left(\tilde{v}_{j}^{-}\right) f_{j} D_{-}^{l}\left(\log f_{j}\right) .
\end{aligned}
$$


Thanks to $(3.27)$, we can express $\tilde{v}_{j}^{+}$and $\tilde{v}_{j}^{-}$with respect to $v_{j}$

$$
\tilde{v}_{j}^{+, s}=v_{j}^{s}+\frac{\Delta v}{2}, \quad \tilde{v}_{j}^{-, s}=v_{j}^{s}-\frac{\Delta v}{2}, \quad s=1,2,3 .
$$

The $3 \times 3$ matrices $S\left(\tilde{v}_{j}^{+}\right)$and $S\left(\tilde{v}_{j}^{-}\right)$then read

$$
S\left(\tilde{v}_{j}^{+}\right)=S\left(v_{j}\right)+\frac{\Delta v^{2}}{4} A+\frac{\Delta v}{2} B\left(v_{j}\right),
$$

and

$$
S\left(\tilde{v}_{j}^{-}\right)=S\left(v_{j}\right)+\frac{\Delta v^{2}}{4} A-\frac{\Delta v}{2} B\left(v_{j}\right),
$$

where $A$ and $B$ stand for the following $3 \times 3$ matrices

$$
A=\left(\begin{array}{ccc}
2 & -1 & -1 \\
-1 & 2 & -1 \\
-1 & -1 & 2
\end{array}\right), \quad B\left(v_{j}\right)=\left(\begin{array}{ccc}
2\left(v_{j_{2}}+v_{j_{3}}\right) & -\left(v_{j_{1}}+v_{j_{2}}\right) & -\left(v_{j_{1}}+v_{j_{3}}\right) \\
-\left(v_{j_{1}}+v_{j_{2}}\right) & 2\left(v_{j_{1}}+v_{j_{3}}\right) & -\left(v_{j_{2}}+v_{j_{3}}\right) \\
-\left(v_{j_{1}}+v_{j_{3}}\right) & -\left(v_{j_{2}}+v_{j_{3}}\right) & 2\left(v_{j_{1}}+v_{j_{2}}\right)
\end{array}\right) \text {. }
$$

Thus, using (3.29) and (3.30), the equality (3.28) becomes

$$
\begin{aligned}
\Delta v^{3} \sum_{j \in \mathbb{Z}^{3}} Q_{\alpha, \beta}(f)\left(v_{j}\right) v_{j_{k}}= & -\frac{\Delta v^{3}}{2} \sum_{j \in \mathbb{Z}^{3}} \sum_{l=1}^{3} \Phi_{k, l}\left(v_{j}\right) f_{j}\left(\left(D_{+}^{l}+D_{-}^{l}\right)\left(\log f_{j}\right)\right) \\
& -\frac{\Delta v^{5}}{8} \sum_{j \in \mathbb{Z}^{3}} \sum_{l=1}^{3} \frac{A_{k, l}}{\left|v_{j}\right|^{3}} f_{j}\left(\left(D_{+}^{l}+D_{-}^{l}\right)\left(\log f_{j}\right)\right) \\
& -\frac{\Delta v^{4}}{4} \sum_{j \in \mathbb{Z}^{3}} \sum_{l=1}^{3} \frac{B_{k, l}\left(v_{j}\right)}{\left|v_{j}\right|^{3}} f_{j}\left(\left(D_{+}^{l}-D_{-}^{l}\right)\left(\log f_{j}\right)\right) .
\end{aligned}
$$

Each term vanishes thanks to arguments of symmetry of $f_{j}$. Thus, provided that $f_{j}$ is symmetric with respect to each coordinate, we proved that the approximation (3.26) of $Q_{\alpha, \beta}(f)$ preserves momentum.

We consider an explicit time discretization and assume the distribution function is known at time $t^{n}$. Then, its value at time $t^{n+1}=t^{n}+\Delta t$ denoted by $\bar{f}_{j}, j \in \mathcal{J}$ (where $\mathcal{J}$ is a bounded discrete set) is given by the following explicit scheme

$$
\bar{f}_{j}=f_{j}+\Delta t Q_{\alpha, \beta}(f)\left(v_{j}\right), \quad \forall j \in \mathcal{J},
$$

where $Q_{\alpha, \beta}(f)\left(v_{j}\right)$ is defined by (3.26) and following [5], $f_{j}$ (which is in factor in $Q_{\alpha, \beta}(f)\left(v_{j}\right)$ ) is approximated by $g_{j}$ given by

$$
g_{j}=\left\{\begin{array}{l}
2 f_{j+1} f_{j} /\left(f_{j+1}+f_{j}\right) \\
2 f_{j-1} f_{j} /\left(f_{j-1}+f_{j}\right)
\end{array}\right.
$$

respectively, according to the discrete operator $D_{+}$or $D_{-}$. We shall determine a condition on the time step $\Delta t$ under which the scheme gives a positive solution for an arbitrary large time. The following proposition sums up this result which can also be found for a more general FokkerPlanck-Landau operator in [5].

Proposition 3.7 There exists a time-sequence $\Delta t_{n}$ such that the scheme (3.32) defines a positive solution at any time (i.e. $\sum_{n} \Delta t_{n}=+\infty$ ).

Proof. Showing that $f_{j}$ does not vanish in finite time is equivalent to prove that

$$
K=\sup _{j \in \mathcal{J}}\left|\frac{f_{j}}{f_{j \pm 1}}\right|
$$


remains bounded. To prove this assertion, we use the following estimate

$$
0 \leq g_{j}=2 \frac{f_{j}}{1+f_{j} / f_{j \pm 1}} \leq 2 f_{j}
$$

and re-write (3.26) as

$$
Q_{\alpha, \beta}(f)\left(v_{j}\right)=-\frac{1}{2}\left(D_{+}^{\star} p_{j}+D_{-}^{\star} q_{j}\right),
$$

where $p_{j}$ and $q_{j}$ are given by

$$
\begin{aligned}
& p_{j}=1 /\left|v_{j}\right|^{3} S\left(\tilde{v}_{j}^{+}\right) f_{j} D_{+}\left(\log f_{j}\right), \\
& q_{j}=1 /\left|v_{j}\right|^{3} S\left(\tilde{v}_{j}^{-}\right) f_{j} D_{-}\left(\log f_{j}\right) .
\end{aligned}
$$

For the sake of simplicity, we restrict ourselves to the discrete operator $D_{+}$and note that a similar technique leads to the same conclusion for $D_{-}$. Hence, using the definitions of $K$ and of $Q_{e i}(f)_{j}$, we have

$$
\left|p_{j}\right| \leq C g_{j} \log (K)
$$

where $C$ is a constant depending on the number of grid points, on the initial condition and on the velocity step $\Delta v$. Indeed, the estimate (3.34) can be deduced from the following bounds

$$
\sup _{j \in \mathcal{J}}\left\|S\left(\tilde{v}_{j}\right)\right\| \leq C,\left|f_{j}\right| \leq C, \forall j \in \mathcal{J}
$$

where $\|\cdot\|$ denotes a matrix norm. Thanks to (3.33), the following inequality holds

$$
\left|\left(D^{\star} p\right)_{j}\right| \leq C \log (K) \sup _{j \in \mathcal{J}} g_{j \pm 1} \leq C \log (K) f_{j} .
$$

We first define $\Delta t_{1}=1 / C \log (K)$ and choose $\Delta t=\alpha \Delta t_{1}$, with $0<\alpha<1$. Then, we obtain

$$
\frac{\bar{f}_{j}}{\bar{f}_{j \pm 1}}=\frac{f_{j}}{f_{j \pm 1}+\Delta t Q_{\alpha, \beta}(f)\left(v_{j \pm 1}\right)}+\Delta t \frac{Q_{\alpha, \beta}(f)\left(v_{j}\right)}{f_{j \pm 1}+\Delta t Q_{\alpha, \beta}(f)\left(v_{j \pm 1}\right)} .
$$

Thanks to the estimates (3.33), (3.35) and (3.36), we have

$$
\frac{\bar{f}_{j}}{\bar{f}_{j \pm 1}} \leq \frac{f_{j}}{f_{j \pm 1}-C \Delta t \log (K) f_{j \pm 1}}+\frac{C \Delta t f_{j} \log (K)}{f_{j \pm 1}-C \Delta t \log (K) f_{j \pm 1}}
$$

Our choice of time step leads to

$$
\frac{\bar{f}_{j}}{\bar{f}_{j \pm 1}} \leq \frac{K(1+\alpha)}{(1-\alpha)}=\beta K
$$

with $\beta>1$. Then, we proceed by induction on $n \in\{0, \ldots, N\}$ and get

$$
\Delta t_{n} \geq n \log (\beta)+\log \left(K_{0}\right),
$$

with $K_{0}=K(0)=\max _{j \in \mathcal{J}}\left\{f_{j}^{0} / f_{j \pm 1}^{0}\right\}<\infty$. Therefore, for the time step $\Delta t_{n}$ defined by $\Delta t_{n}=$ $\alpha / C \log \left(K_{n}\right)$, the solution is nonnegative and we get the following estimate

$$
\Delta t_{n} \geq \frac{\alpha}{C\left(n \log (\beta)+\log \left(K_{0}\right)\right)} .
$$

The right hand side of this inequality is a non convergent series and thus

$$
t^{n}=\sum_{k \leq n} \Delta t_{k} \underset{n \rightarrow+\infty}{\longrightarrow}+\infty
$$




\subsection{Approximation to the Landau operator}

In this section, we shall consider the discretization of the $\alpha$ - $\alpha$ collision operator, i.e. the nonlinear Fokker-Planck-Landau operator (1.3) in the whole $3 D$ velocity space. As in the previous section, we restrict ourselves to the space homogeneous case. More precisely, we are concerned with the numerical approximation of the FPL equation

$$
\frac{\partial f}{\partial t}=Q(f, f)
$$

where $Q(f, f)$ is given by (1.3). As we described before, it is convenient to write the FPL operator in its weak form. To that purpose, we first define for any test function $\varphi(v)$ sufficiently smooth,

$$
\begin{aligned}
& G\left(v, v^{\prime}\right)= \\
& \quad-\frac{1}{2} f(t, v) f\left(t, v^{\prime}\right)\left[\nabla_{v} \varphi(v)-\nabla_{v^{\prime}} \varphi\left(v^{\prime}\right)\right]^{T} \Phi\left(v-v^{\prime}\right)\left[\nabla_{v} \log (f(t, v))-\nabla_{v^{\prime}} \log \left(f\left(t, v^{\prime}\right)\right)\right] .
\end{aligned}
$$

Then, we write the FPL operator using the weak formulation

$$
\int_{\mathbf{R}^{3}} Q(f, f)(v) \varphi(v) d v=\int_{\mathbf{R}^{3} \times \mathbf{R}^{3}} G\left(v, v^{\prime}\right) d v^{\prime} d v .
$$

We now recall the basic entropy conservative discretization introduced in [15], using the weak form (3.38) we define $Q(f, f)_{j}$ as an approximation of $Q(f, f)\left(v_{j}\right)$ such that

$$
\sum_{j \in \mathbb{Z}^{3}} Q(f, f)_{j} \varphi_{j} \Delta v^{3}=\sum_{(j, m) \in \mathbb{Z}^{6}} G\left(v_{j}, v_{m}\right) \Delta v^{6}
$$

the value $G\left(v_{j}, v_{m}\right)$ is defined for any test sequence $\varphi$ by

$$
G\left(v_{j}, v_{m}\right)=-\frac{1}{2} f_{j}(t) f_{m}(t)\left[D \varphi_{j}-D \varphi_{m}\right]^{T} \Phi\left(v_{j}-v_{m}\right)\left[D(\log f(t))_{j}-D(\log f(t))_{m}\right],
$$

where $D$ is again a finite difference operator approximating the usual gradient operator $\nabla_{v}$ (see section 3.2).

We denoted by $f_{j}(t)$ the value of the approximated distribution function at velocity $v_{j}$ and time $t$. From the weak formulation, the evolution of this discretized function is then governed by the following system of differential equations

$$
\frac{d f_{j}(t)}{d t}=Q(f, f)_{j}(t)=\left(D^{*} p(t)\right)_{j}, \quad j \in \mathbb{Z}^{3},
$$

where $D^{*}$ is the formal adjoint of the finite difference operator $D$, and

$$
p_{j}(t)=\Delta v^{3} \sum_{m \in \mathbb{Z}^{3}} f_{j}(t) f_{m}(t) \Phi\left(v_{j}-v_{m}\right)\left(D(\log f(t))_{j}-D(\log f(t))_{m}\right) .
$$

The FPL operator is finally approximated by the average of the discrete operators obtained from the down-wind and up-wind finite difference operators $D$. From the duality relation (3.39), it is an easy matter to verify that the discrete model preserves mass, momentum and energy. Furthermore, taking $\varphi=\log f$ in (3.39) leads to the entropy inequality at the discrete level. Finally, an uncentered approximation of the gradient ensures that the only equilibrium states are discrete Maxwellians. As observed in the previous subsection, the use of centered discrete operator leads to non conserved quantities. In [7], the authors then re-write this scheme as the sum of a second order approximation and an artificial viscosity term in $\Delta v^{2}$ which kills spurious conservations. Nevertheless, a direct implementation of (3.40) remains too expensive. Several algorithms have been proposed to treat the computational cost issue (see [7] and [8]). Here, the multigrid method 
is employed. For the details of this method applied to the discretization of the FPL operator, we refer the reader to [7] and [8].

For the time discretization of (3.40), an Euler explicit scheme is used. From [5], the positivity of the distribution function and the decrease of the entropy are ensured under a condition on the time step $\Delta t$. This condition is similar to the condition derived in Proposition 3.7, but it is possible to avoid it by using a semi-implicit method [27].

\section{Numerical simulations}

\subsection{Numerical results of the linear collision operator}

We present some numerical results to test the ability of the discretization (3.26)-(3.27) and then consider the space homogeneous equation (3.21). The initial datum is chosen to be bi-Maxwellian, i.e. a sum of two Maxwellian functions

$$
f_{0}(x, v)=\frac{1}{2}\left(\mathcal{M}_{1, v_{1}, 1}(v)+\mathcal{M}_{1, v_{2}, 1}(v)\right)
$$

where $\mathcal{M}_{\rho, u, T}$ is given by $(2.1)$, and

$$
v_{1}=(-1,0,0), \quad v_{2}=(1,0,0) .
$$

This test is performed with $v_{\max }=7$ and with different numbers of points in velocity $N_{v}=20,40$ and 60 .

We compute an approximation of each component of the temperature $T_{\alpha}$, for $\alpha \in\{x, y, z\}$

$$
T_{\alpha}(t)=\sum_{j}\left(v_{j}^{\alpha}-u^{\alpha}(t)\right)^{2} f_{j}(t) \Delta v^{3}
$$

where $v_{j}^{\alpha}$ and $u^{\alpha}(t)$ respectively denote the $\alpha$-th component of $v_{j}$ and of the mean velocity $u(t)$. We are also interested in the approximation of the kinetic entropy $H(t)$

$$
H(t)=\sum_{j} f_{j}(t) \log f_{j}(t) \Delta v^{3} .
$$

On Figure 1, we represent the time relaxation of the components of the temperatures to their final value $T$, which corresponds in this case to the initial temperature since momentum is initially zero. We observe that the steady state is reached at about time $t=25$ for the different approximations. Since our algorithm preserves exactly mass, momentum and energy, the steady state is well approximated.

Now, on Figure 2, we represent the evolution of the kinetic entropy for different numbers of points in velocity. The entropy is well decreasing in time and has first a fast decay behavior and next slowly converges to the equilibrium. The three different approximations give the same steady state which corresponds to the kinetic entropy of the Maxwellian associated to the initial mass, momentum and energy $\mathcal{M}_{1,0,4 / 3}(v)$.

\subsection{Linear Landau damping}

We now consider the full Fokker-Planck-Landau equation (1.1)-(1.2) where the collision operators are given by (1.3) and (2.4) (with $\rho_{\beta}=1$ and $u_{\beta}=0$ ). The initial condition is chosen as a perturbation of the global equilibrium

$$
f_{0}(x, v)=\frac{1}{(2 \pi)^{3 / 2}} \exp \left(-|v|^{2} / 2\right)(1+A \cos (k x)), \quad(x, v) \in[0,2 \pi / k] \times \mathbb{R}^{3},
$$




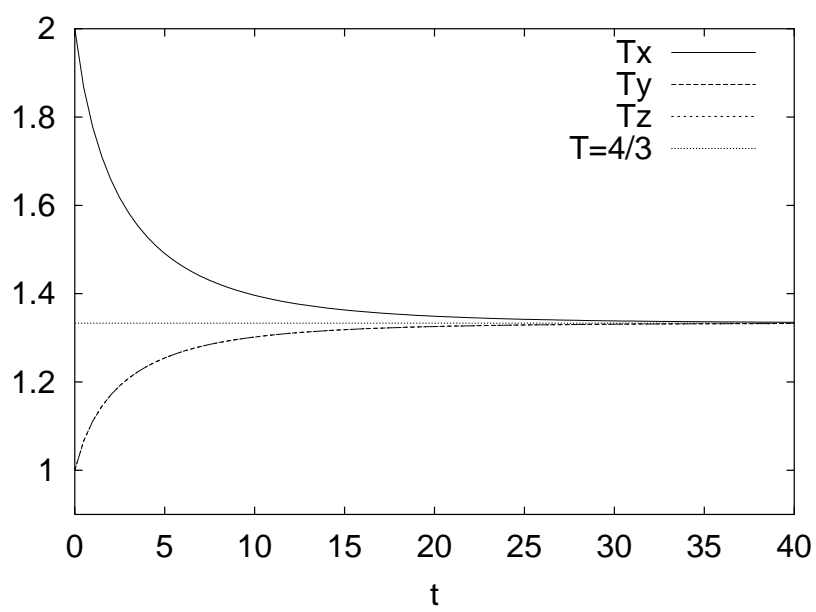

Figure 1: Components of the temperature as a function of time. The temperature of the Maxwellian associated to the initial condition is also plotted for comparison.

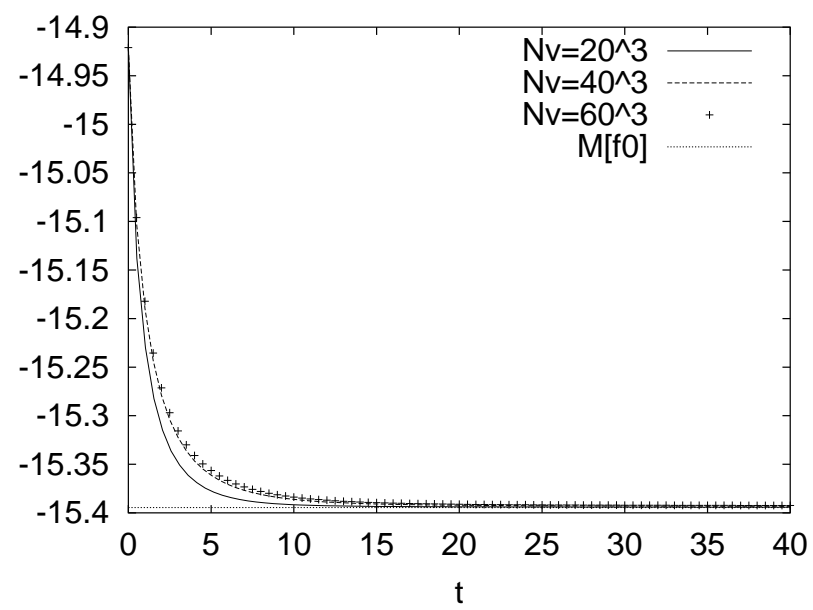

Figure 2: Kinetic entropy as a function of time. The entropy of the Maxwellian associated to the initial condition is also plotted for comparison (dashed line). 
where $A$ is the amplitude of the perturbation and $k$ denotes the wave number. In this subsection, $A$ is taken small enough $\left(A=10^{-5}\right)$ such that we can consider linear regimes.

To capture the Landau damping, the size of the velocity domain must be chosen greater than the phase velocity $v_{\phi}$, which corresponds to the singularity point in the dispersion relation. The phase velocity is given by $v_{\phi}=\omega / k$, where $\omega$ is the frequency related to $k$ and approximated by

$$
\omega^{2}=1+3 k^{2} .
$$

Then, we set $v_{\max }=5.75$ where the velocity grid extends from $-v_{\max }$ to $v_{\max }$. We use a number of cells $N_{v}=32$ points in each direction of the velocity and $N_{x}=50$ in the one dimensional spatial direction. The boundary conditions for the distribution function are periodic in the physical space whereas the distribution function is truncated to zero for large velocities.

In this test, we are interested in the evolution of the square root of the electric energy approximated by

$$
\mathcal{E}_{h}(t)=\left(\sum_{i} \Delta x E_{i}^{2}(t)\right)^{1 / 2} .
$$

Indeed, according to the Landau theory, the amplitude of $\mathcal{E}_{h}(t)$ is expected to be exponentially decreasing with a frequency $\omega$.

On Figure 3, we first represent the evolution of $\mathcal{E}_{h}(t)$ in logarithm scale, where the wave number is fixed to $k=0.3$ and different collision frequencies are taken i.e. $\nu=0,0.01$ and 0.05 . We observe that the amplitude of $\mathcal{E}_{h}(t)$ is damped exponentially in time as predicted by the Landau theory. Moreover, the influence of collisions on the electric energy is well reproduced since the increasing of the collision frequency $\nu$ induces a stronger damping rate. In conclusion, collisions play an additional role in the damping of the electric energy and its amplitude seems to be always exponentially decreasing in time.

Then, we study on Figures 4 and 5 the influence of the wave number $k$ on the evolution of the electric energy and consider $k=0.2,0.3,0.4$ and 0.5 . On the one hand, the collision frequency is set to zero. We observe that $\mathcal{E}_{h}(t)$ is always exponentially decreasing, where the damping rate is increasing when $k$ becomes larger (see Figure 4). On the other hand, we choose $\nu=0.01$ and plot the evolution of $\mathcal{E}_{h}(t)$ on Figure 5 . The behavior of the electric energy amplitude is still exponential and depends on the value of $k$ as in the previous case. Besides, we notice that the damping is more important than in the collisionless case.

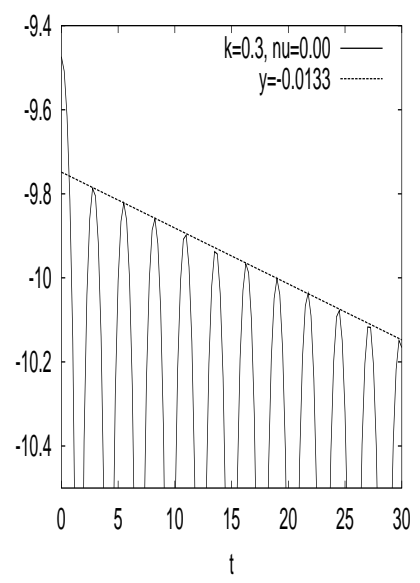

(a)

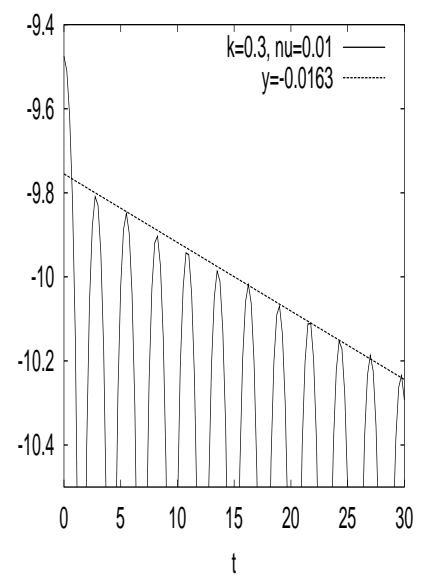

(b)

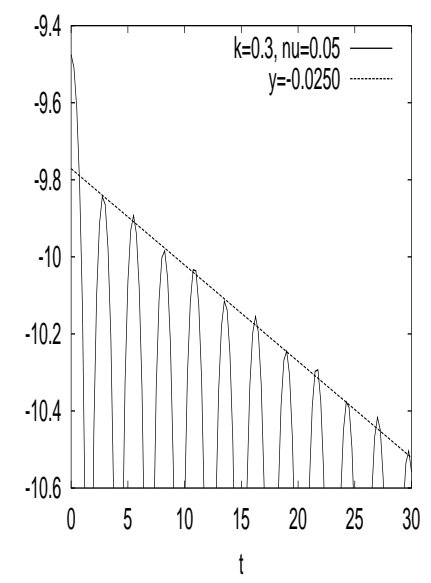

(c)

Figure 3: Study of the influence of the collisional parameter $\nu . k=0.3$ and $\nu=0,0.01,0.05$. 


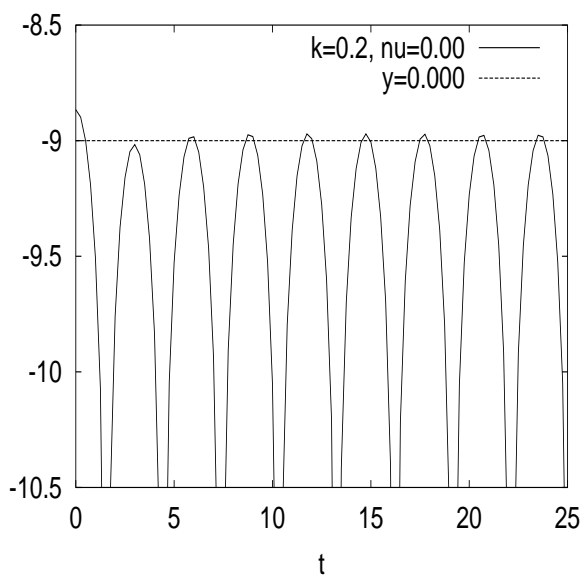

(a)

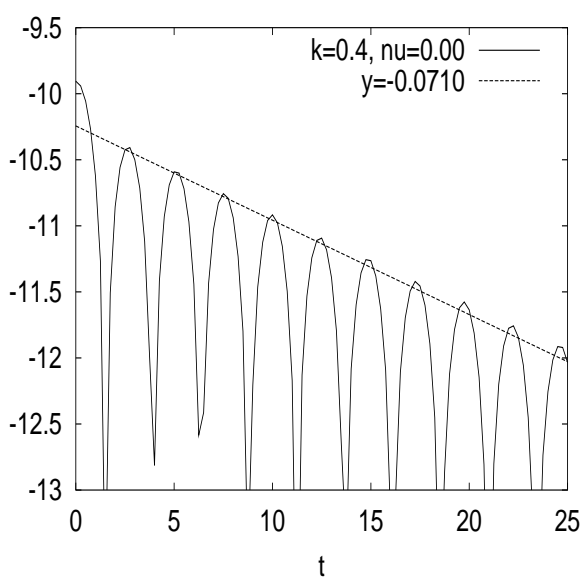

(c)

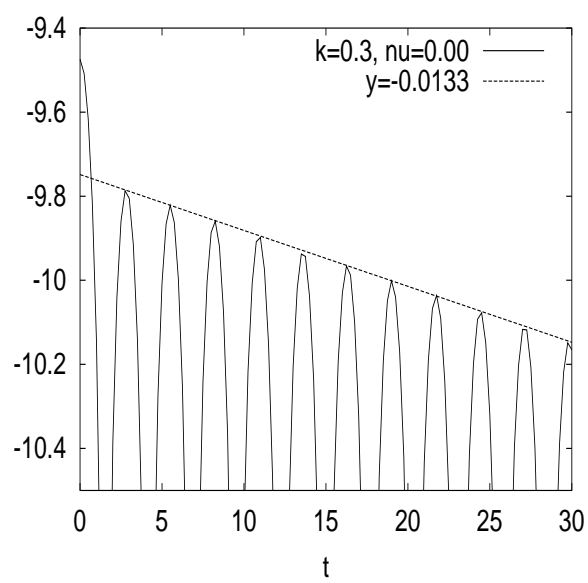

(b)

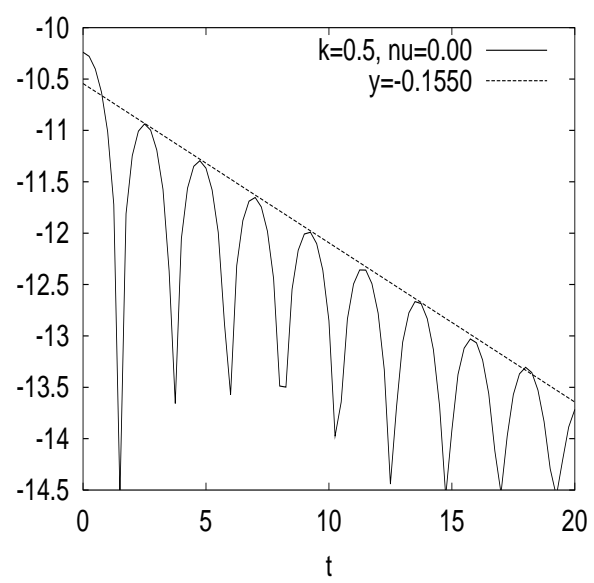

(d)

Figure 4: Study of the influence of $k$ in the non-collisional case $(\nu=0) . k=0.2,0.3,0.4,0.5$. 


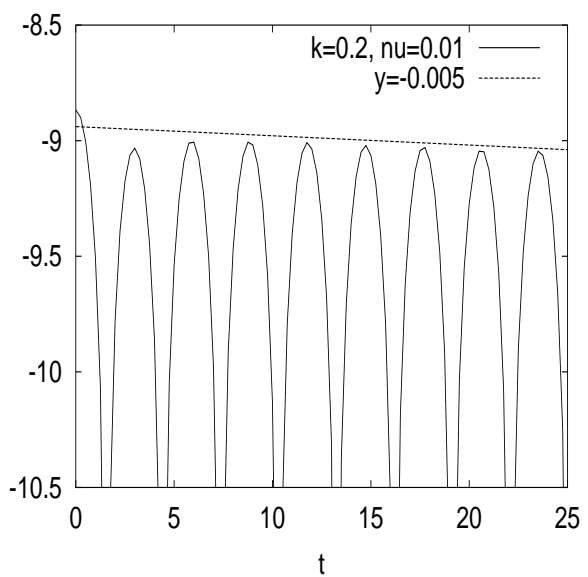

(a)

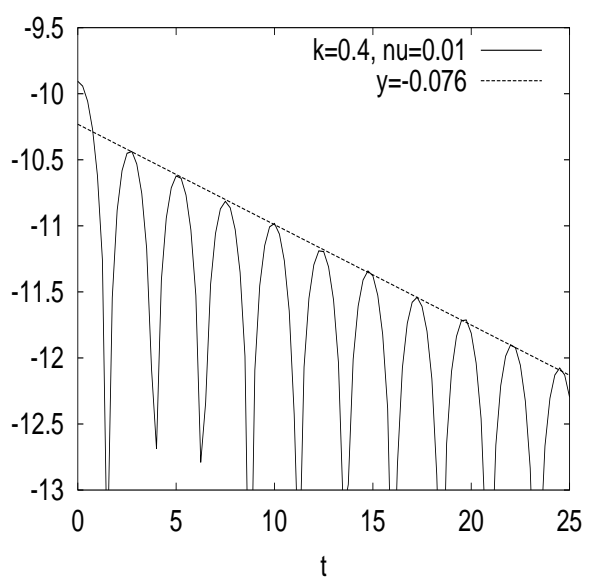

(c)

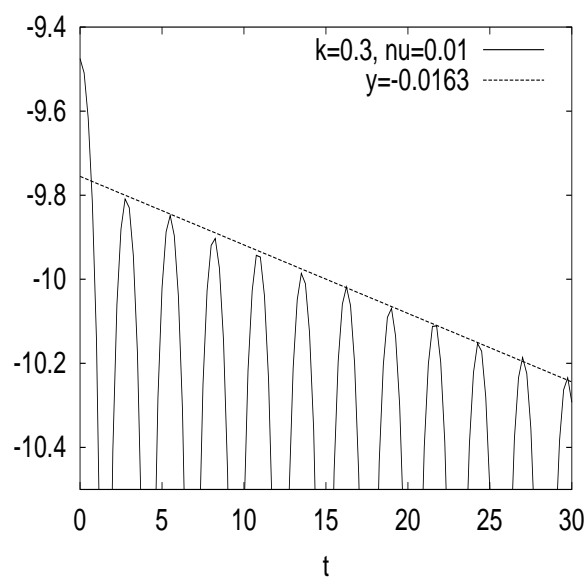

(b)

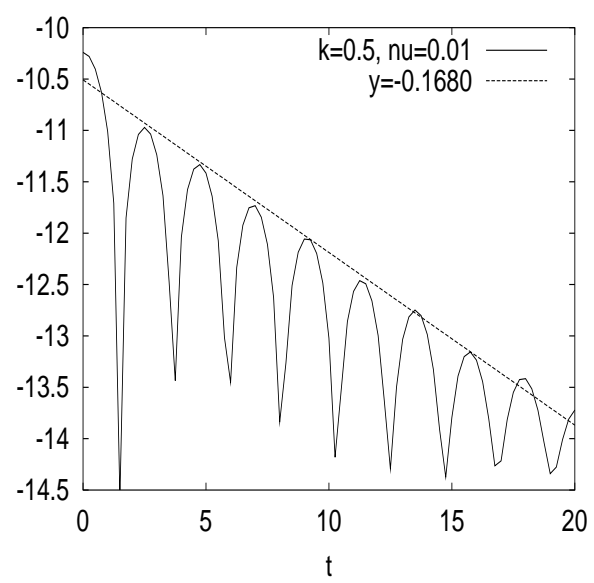

(d)

Figure 5: Study of the influence of $k$ in the collisional case $(\nu=0.01) . k=0.2,0.3,0.4,0.5$. 


\begin{tabular}{|c|c|c|c|}
\hline & Estimates [10,16] & Estimates [22] & Numerical \\
\hline$k=0.2$ & $-7.10^{-5}$ & $-6.10^{-5}$ & 0 \\
\hline$k=0.3$ & -0.020 & -0.0132 & -0.0133 \\
\hline$k=0.4$ & -0.096 & $\mathrm{X}$ & -0.071 \\
\hline$k=0.5$ & -0.151 & $\mathrm{X}$ & -0.155 \\
\hline
\end{tabular}

Table 1: Comparison of theoretical and numerical damping coefficients $\gamma$ with $\nu=0$.

These numerical results can be compared to theoretical estimates on the damping rate and frequency. Indeed, when the amplitude of the perturbation $A$ is small enough, we can linearize the initial model and solve the dispersion relation to evaluate the damping plasma wave and the frequency $\omega$. In the collisionless case, the theoretical damping coefficient is often estimated by (see $[10,16])$

$$
\gamma_{L}=-\sqrt{\frac{\pi}{8}} \frac{1}{k^{3}} \exp \left(-1 /\left(2 k^{2}\right)-3 / 2\right) .
$$

In the collisional case, the theoretical damping reads (see $[10,16]$ )

$$
\gamma=\gamma_{L}+\gamma_{C}, \quad \text { with } \gamma_{C}=-\frac{1}{3} \nu \sqrt{2 / \pi},
$$

where $\gamma_{L}$ is given by (4.3). In Tables 1 and 2, we compare the damping rates (4.3), (4.4) for different electrical waves in both collisional and collisionless cases, with the damping computed from our numerical method. We also compare in Table 3 the oscillation frequencies obtained numerically and computed theoretically through (4.2).

Our numerical results correctly agree with (4.2), (4.3) and (4.4) in a qualitative sense. Indeed, exponential damping behavior is well obtained and the influence of collisions on the damping rate is also recovered (see Figures 4 and 5). More precisely, when $k=0.5$ our results are in very good agreement with the theoretical estimates, but for smaller wave numbers the comparison is not satisfying. However it is known that for small $k$, estimates (4.2) and (4.3) are not really accurate because the expansion of the dispersion relation is not accurate enough. Then, we use more accurate formulas given in [22], which are more correct for small wave numbers

$$
\gamma_{L}=-\sqrt{\frac{\pi}{8}}\left(\frac{1}{k^{3}}-6 k\right) \exp \left(-1 /\left(2 k^{2}\right)-3 / 2-3 k^{2}-12 k^{4}\right)
$$

and the frequency $\omega$ is

$$
\omega^{2}=1+3 k^{2}+6 k^{4}+24 k^{6} .
$$

In this case, the numerical results for $k$ smaller than 0.3 perfectly agree with the relation (4.5). In the collisional case, it is expected that the damping rate does not depend on $k$, but only on the collision frequency $\nu$. The damping rates are reported in Table 2 for different wave numbers $k$ and $\nu=0.01$; the damping rates obtained from the numerical method are weakly dependent on the wave number $k$ (when it is small enough), but do not correspond exactly to the estimated value (4.4).

Finally, the numerical values of the frequency $\omega$ correctly agree with formula (4.2) and when $k$ is small with (4.6). The behavior of $\omega$ with respect to $k$ is also consistent with the theoretical values.

\subsection{Nonlinear Landau damping}

In this subsection, we also consider the Fokker-Planck-Landau equation (1.1)-(1.2) where the collision operators are given by (1.3) and (2.4) (with $\rho_{\beta}=1$ and $u_{\beta}=0$ ). The initial condition is chosen as in the previous subsection, but the perturbation $A$ is now larger. Hence, we do not 


\begin{tabular}{|c|c|c|c|}
\hline & Estimates [10, 16] & Estimates [22] & Numerical \\
\hline$k=0.2$ & -0.003 & -0.003 & -0.004 \\
\hline$k=0.3$ & -0.023 & -0.0167 & -0.0163 \\
\hline$k=0.4$ & -0.0987 & $\mathrm{X}$ & -0.0825 \\
\hline$k=0.5$ & -0.154 & $\mathrm{X}$ & -0.168 \\
\hline
\end{tabular}

Table 2: Comparison of theoretical and numerical damping coefficients $\gamma$ with $\nu=0.01$.

\begin{tabular}{|c|c|c|c|}
\hline & Estimates [10, 16] & Estimates [22] & Numerical \\
\hline$k=0.2$ & 1.0583 & 1.0635 & 1.0694 \\
\hline$k=0.3$ & 1.1269 & 1.1559 & 1.1615 \\
\hline$k=0.4$ & 1.2165 & $\mathrm{X}$ & 1.2822 \\
\hline$k=0.5$ & 1.3228 & $\mathrm{X}$ & 1.3962 \\
\hline
\end{tabular}

Table 3: Comparison of theoretical and numerical frequency $\omega$.

consider linear regimes and the Landau theory cannot be applied. Then, we cannot compare our results to theoretical estimates but some authors already studied this test in both collisional [21] and collisionless cases $[28,36]$.

To describe nonlinear effects, we have to consider a velocity set of size $v_{\max }=7$ in each direction. We use a number of cells $N_{v}=32$ in velocity space whereas we consider $N_{x}=50$ points in physical space. On Figures 6 and 7, we represent the evolution of the discrete electric energy in logarithm scale, with two different values of $A$.

On Figure $6, A$ is taken equal to 0.1 whereas the excited mode $k$ is equal to 0.3 . We consider the collisionless case $(\nu=0)$ and a collisional regime with $\nu=0.01$. As in the previous subsection, the electric energy is damped exponentially and when the collision frequency increases, the damping of the electric energy is stronger. Moreover, we notice that the damping is much stronger than in the linear context in both cases. It seems that the Landau theory is robust with respect to the initial perturbation since in this case the initial datum is strongly perturbed and the decay of the amplitude of the electric energy remains exponential in time.

On Figure 7 , the perturbation is more important $(A=0.2)$ whereas $k$ is still equal to 0.3 . Then, we compare the evolution of the electric energy for three different values $\nu=0,0.01$ and 0.05. In the collisionless regime, the results are in good agreement with the simulations presented in the literature: the electric energy is first exponentially decreasing and is next oscillating around a constant. At variance, in presence of collisions $(\nu \neq 0)$, these oscillations are soften. Moreover, when the parameter $\nu$ is growing up $(\nu=0.05)$, the amplitude of the electric field decreases in time, as observed in [21]. Let us pointed out that at the beginning of the simulation a damped exponential behavior is observed as in the linear case, but the damping coefficient is more important. We also plot the isovalues of the distribution function

$$
F\left(t, x, v_{x}\right)=\int_{\mathbf{R}^{2}} f\left(t, x, v_{x}, v_{y}, v_{z}\right) d v_{y} d v_{z}
$$

at time $t=0,2.5,5$ and 7.5. Without collisions, some particles are trapped around the phase velocity $v_{\phi}=\omega / k \simeq 3.84$ generating a bump, which propagates in the phase space [21]. However, when the collision frequency is increasing the electric energy is damped and few particles are trapped (see Figure 8).

Besides, when the global equilibrium is strongly perturbed, slope correctors act to ensure the positivity of $f$ and then the total energy is not exactly preserved, as discussed in section 3.1. Then, we study the variations of the total energy on Figure 9 for $A=0.2$. As expected, we observe that the total energy is not conserved. Nevertheless, we notice that the variations are relatively small (less than $3 \%$ ). Moreover, these variations are small compared to the variations of the total energy 
given by others numerical methods (semi-Lagrangian schemes).

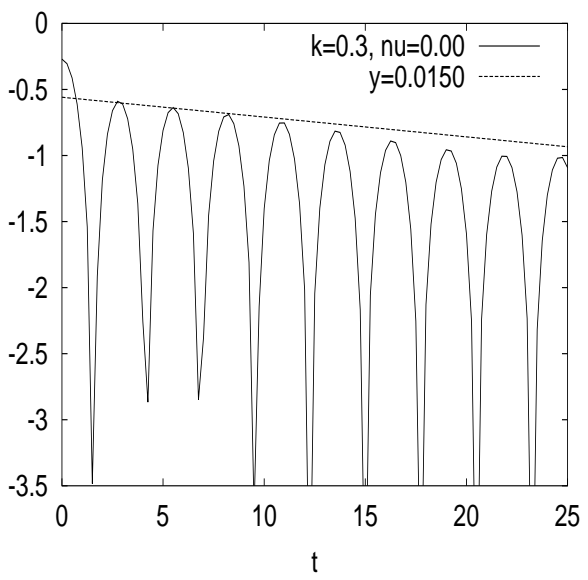

(a)

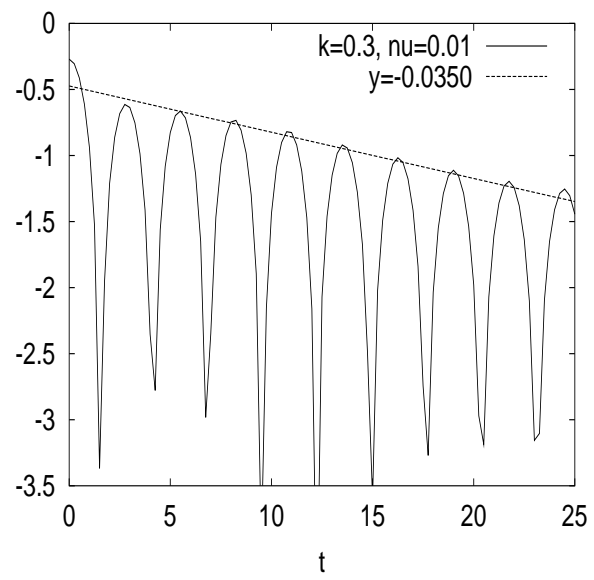

(b)

Figure 6: Study of the influence of the collisional parameter $\nu$ in the non linear case $A=0.1$, $k=0.3$ with (a) $\nu=0$ and (b) $\nu=0.01$.

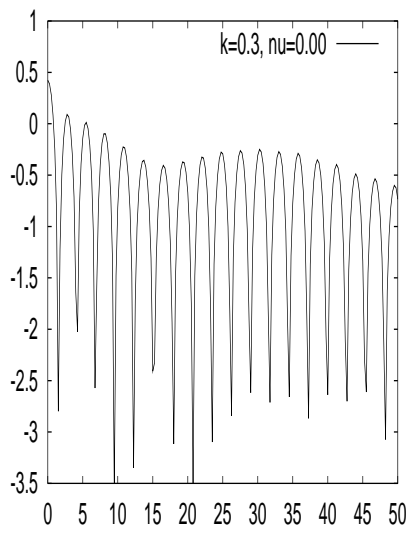

(a)

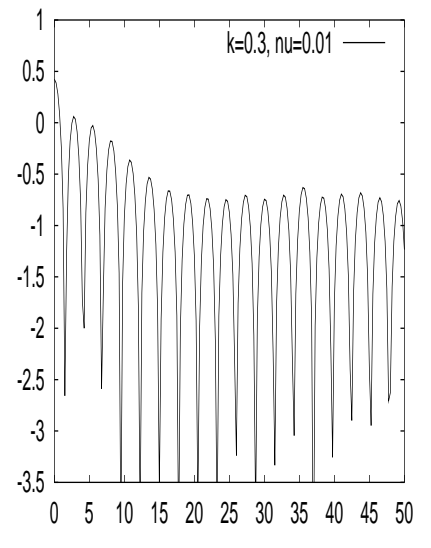

(b)

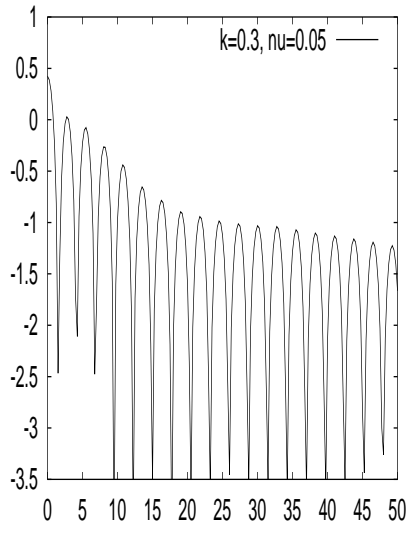

(c)

Figure 7: Study of the influence of the collisional parameter $\nu$ in the non linear case $A=0.2$, $k=0.3$ with (a) $\nu=0$, (b) $\nu=0.01$ and (c) $\nu=0.05$.

\section{Conclusion}

We have developped a new numerical scheme for realistic and collisional plasmas in one dimension in the physical space and in three dimensions in velocity space. The method takes into account two different species of particles (e.g. electrons and ions). On the one hand, a new discretization of the Vlasov-Poisson equation has been proposed. For this approximation, we proved conservation of mass and total energy. On the other hand, a discretization of the electron-ion collision operator is derived respecting the main properties of the continuous operator (conservation of mass, momentum, energy and decrease of the entropy). In the context of the Landau damping, our results are in a very good agreement with the theoretical results available in the literature. Furthermore, 

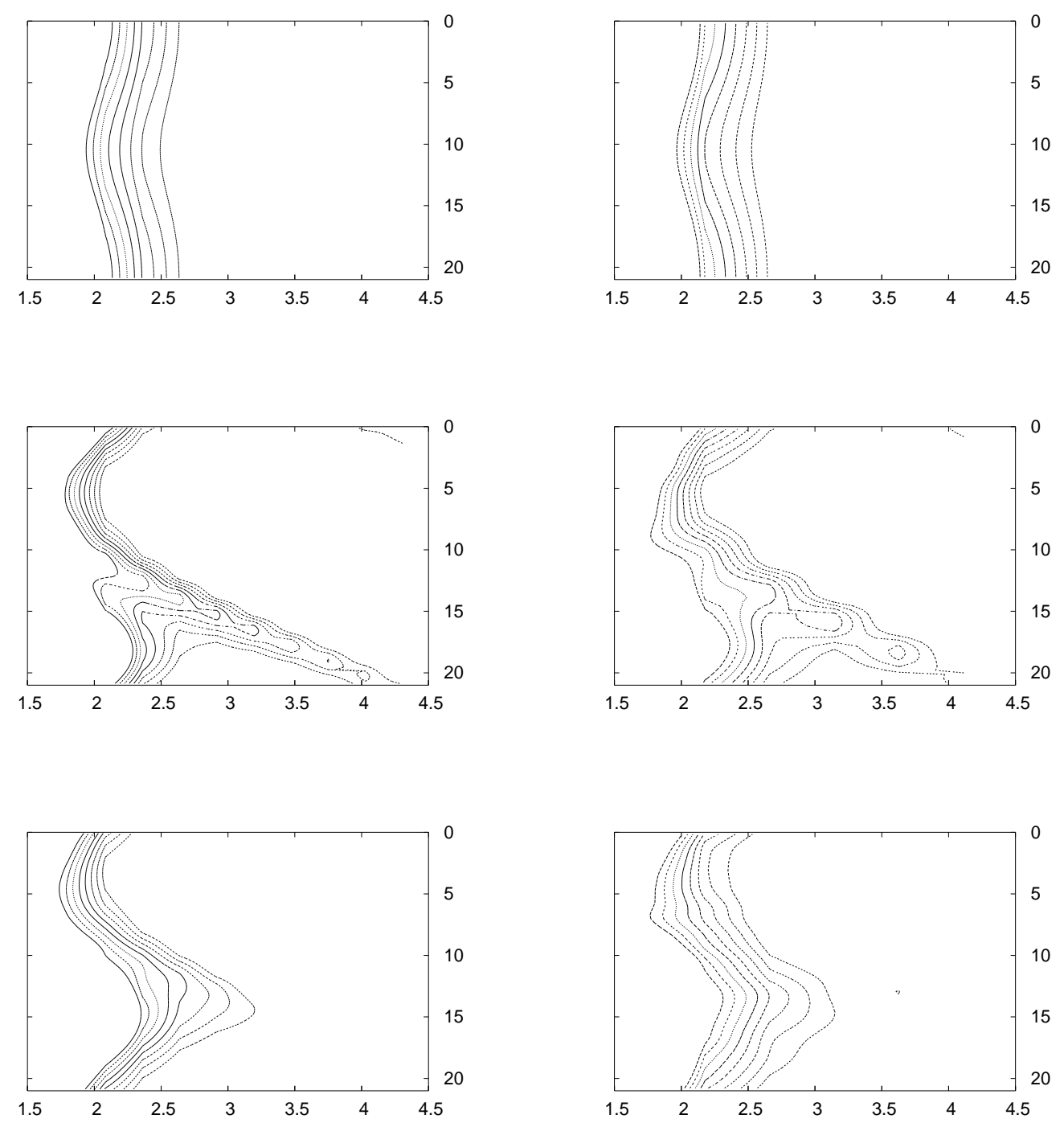

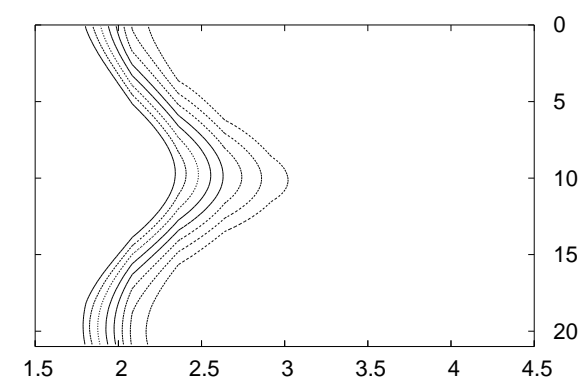

(a)

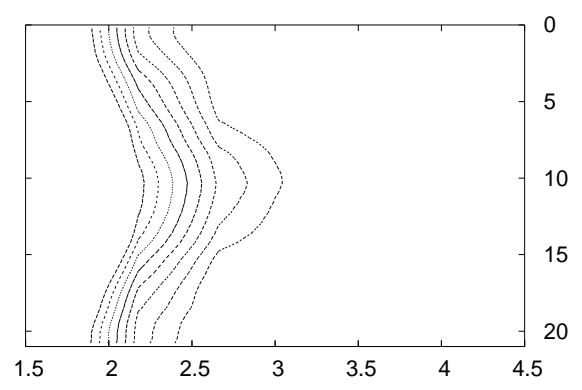

(b)

Figure 8: Isovalues (from 0.015 to 0.050 ) of the projection of the distribution function on the $x-v_{x}$ plane with $A=0.2, k=0.3$ and (a) $\nu=0$, (b) $\nu=0.01$. 


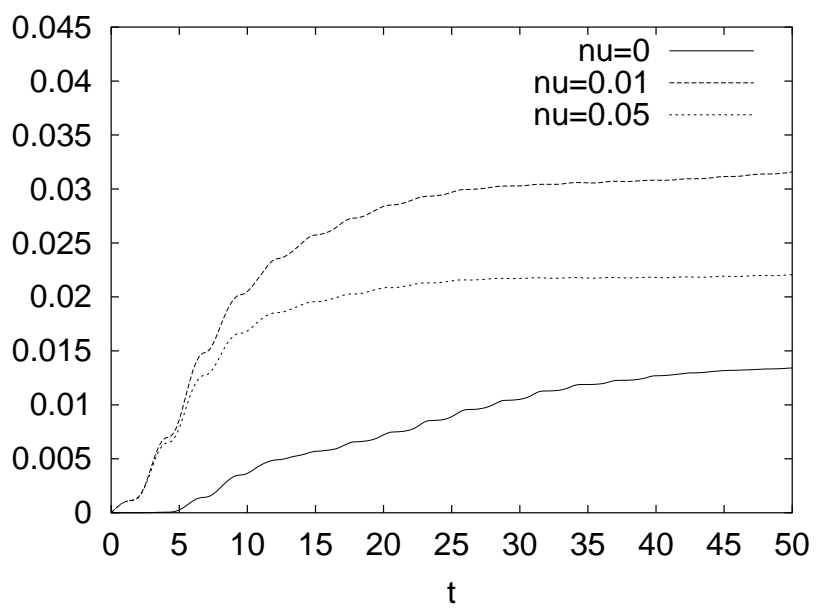

Figure 9: Evolution of the total energy in both collisional and collisionless case, in the non linear case $A=0.2 . k=0.3$ with $\nu=0,0.01$ and 0.05 .

for strong perturbations we observe the effect of collisions on the damping of the electric energy and on the long time behavior of the solution.

Several extensions and applications of this work can be considered. For instance, the ions density and mean velocity can be governed by kinetic or hydrodynamic equations coupled with the description of the electrons. Moreover, the effect of a self-consistent and applied magnetic field can be also investigated.

Acknowledgements: Helpful discussions on numerical and modeling issues with Bruno Dubroca, Vladimir Tickonchuk and Mohammed Lemou are gratefully acknowledged. This work was also partially supported by the European network HYKE, funded by the EC as contract HPRN-CT-2002-00282.

\section{References}

[1] A. A. Arsen'ev, O. E. Buryak, On the connection between a solution of the Boltzmann equation and a solution of the Fokker-Planck-Landau equation, Math. USSR Sbornik, 69, 465-478, (1991).

[2] Yu. A. Berezin, V. N. Khudick, M. S. Pekker, Conservative finite difference schemes for the FokkerPlanck equation not violating the law of an increasing entropy, J. Comput. Phys., 69, 163-174, (1987).

[3] C. K. Birdsall and A. B. Langdon, Plasma Physics via Computer Simulation, Institute of Physics Publishing, Bristol and Philadelphia, (1991).

[4] A. V. Bobylev, I. F. Potapenko, V. A. Chuyanov, Kinetic equations of the Landau type as a model of the Boltzmann equation and completely conservative difference schemes, U.S.S.R. Comput. Maths. Math. Phys., 20, 190-201, (1981).

[5] C. Buet, S. Cordier, Numerical analysis of conservative and entropy schemes for the Fokker-PlanckLandau equation, SIAM J. Numer. Anal., 36, 953-973 (1999).

[6] C. Buet, S. Cordier, Conservative and entropy decaying numerical scheme for the isotropic FokkerPlanck-Landau equation, J. Comput. Phys., 145, 228-245, (1998).

[7] C. Buet, S. Cordier, P. Degond, M. Lemou, Fast algorithms for numerical, conservative and entropy approximations of the Fokker-Planck-Landau equation, J. Comput. Phys., 133, 310-322 (1997). 
[8] C. Buet, S. Cordier, F. Filbet, Comparison of Numerical schemes for Fokker-Planck-Landau equation, ESAIM Proc. 10, 161-181, (1999)

[9] C. Buet, S. Dellacherie, R. Sentis, Numerical solution of an ionic Fokker-Planck equation with electronic temperature, SIAM J. Numer. Anal., 39, 1219-1253, (2001).

[10] F. F. Chen, Introduction to Plasma Physics and controlled fusion, 2nd edition, Plenum Press, New York and London.

[11] S. Dellacherie, Numerical resolution of an ion-electron collision operator in axisymmetrical geometry. Special issue comprised of papers presented at the Conference on Asymptotic and Numerical Methods for Kinetic Equations (Oberwolfach, 2001), Transport Theory Statist. Phys., 31, 397-429, (2002).

[12] S. Dellacherie, Contribution à l'analyse et à la simulation numériques des équations cinétiques décrivant un plasma chaud, PhD Thesis, Paris 7, (1998).

[13] A. De Coster, B. Perthame, P. Marcowich, Modeling of Collisions, Series in Applied Mathematics, (1998).

[14] P. Degond, B. Lucquin-DesReux, The Fokker-Planck asymptotics of the Boltzmann collision operator in the Coulomb case, M3AS, 2, 167-182, (1992).

[15] P. Degond, B. Lucquin-Desreux, An entropy scheme for the Fokker-Planck collision operator of plasma kinetic theory, Numer. Math. 68, 239-262 (1994).

[16] J. P. Delcroix, A. Bers, Physique des plasmas, Savoirs Actuels, InterEditions, CNRS Editions, (1994).

[17] L. Desvillettes, On asymptotics of the Boltzmann equation when the collisions become grazing, Transp. Theory Stat. Phys., 21, 259-276, (1992).

[18] E. M. Epperlein, Implicit and conservative difference schemes for the Fokker-Planck equation, J. Comp. Phys., 112, 291-297, (1994).

[19] F. Filbet, E. SonnendrüCker, P. Bertrand, Conservative Numerical schemes for the Vlasov equation, J. Comput. Phys., 172, 166-187 (2001).

[20] F. Filbet and E. Sonnendrücker, Comparison of Eulerian Vlasov solvers, Comput. Phys. Communications, 151, 247-266, (2003).

[21] F. Filbet and L. Pareschi, Numerical method for the accurate solution of the Fokker-Planck-Landau equation in the non homogeneous case, J. Comput. Phys. 179, 1-26, (2002).

[22] C. J. McKinstrie, R. E. Giacone, E. A. Startsev, Accurate formulas for the Landau damping rates of electrostatic waves, Physics of Plasmas, 6, 463-466, (1999).

[23] R. B. Horne and M. P. Freeman, A new code for electrostatic simulation by numerical integration of the Vlasov and Amp'ere equations using MacCormack's method, J. Comput. Phys., 171, 182-200, (2001).

[24] L. D. Landau, Die kinetische gleichung für den fall Coulombscher vechselwirkung, Phys. Z. Sowjet., 154, (1936). Trad. The transport equation in the case of the Coulomb interaction in D.ter Haar Ed. Collected papers of L. D. Landau, 163-170. Pergamon press, Oxford (1981).

[25] M. Lemou, Multipole expansions for the Fokker-Planck-Landau operator, Numer. Math., 78, 597-618, (1998).

[26] M. Lemou, Numerical algorithms for axisymmetric Fokker-Planck-Landau operators, J. Comput. Phys., 157, 762-786, (2000).

[27] M. Lemou and L. Mieussens, Fast implicit schemes for the Fokker-Planck-Landau equation, in preparation.

[28] G. Manfredi, Long time behaviour of the non linear Landau damping, Phys. Rev. Letters, 79, 2815-2818, (1997).

[29] K. Nanbu and S. Yonemura, Weighted particles in Coulomb collision simulations based on the theory of a cumulative scattering angle, J. Comp. Phys., 145, 639-654, (1998).

[30] L. Pareschi, G. Russo and G. Toscani, Fast spectral methods for Fokker-Planck-Landau collision operator, J. Comput. Phys., 165, 216-236, (2000).

[31] M. S. Pekker and V. N. Khudik, Conservative difference schemes for the Fokker-Planck equation, U.S.S.R. Comput. Maths. Math. Phys., 24, 206-210, (1984). 
[32] I. F. Pota penko and C. A. de Arzevedo, The completely conservative difference schemes for the nonlinear Landau-Fokker-Planck equation, J. Comput. Appl. Math., 103, 115-123, (1999).

[33] M. N. Rosenbluth, W. MacDonald and D. L. Judd, Fokker-Planck equation for an inverse square force, Phys. Rev., 107, 1-6, (1957).

[34] M. Shoucri and G. Knorr, Numerical integration of the Vlasov equation, J. Comput. Phys., 14, 84-92, (1974).

[35] C. Villani, A review of mathematical topics in collisional kinetic theory, Handbook of Fluid Mechanics, S. Frielander and D. Serre, Editors, (2003).

[36] Т. Zhоu, Y. Guo and C.-W. Shu, Numerical study on Landau damping, Physica D, 157, 322-333, (2001). 